TRANSACTIONS OF THE

AMERICAN MATHEMATICAL SOCIETY

Volume 355, Number 8 , Pages 3009-3046

S 0002-9947(03)03294-X

Article electronically published on April 24, 2003

\title{
HOMOLOGY OF PSEUDODIFFERENTIAL OPERATORS ON MANIFOLDS WITH FIBERED CUSPS
}

\author{
ROBERT LAUTER AND SERGIU MOROIANU
}

\begin{abstract}
The Hochschild homology of the algebra of pseudodifferential operators on a manifold with fibered cusps, introduced by Mazzeo and Melrose, is studied and computed using the approach of Brylinski and Getzler. One of the main technical tools is a new convergence criterion for tri-filtered half-plane spectral sequences. Using trace-like functionals that generate the 0dimensional Hochschild cohomology groups, the index of a fully elliptic fibered cusp operator is expressed as the sum of a local contribution of Atiyah-Singer type and a global term on the boundary. We announce a result relating this boundary term to the adiabatic limit of the eta invariant in a particular case.
\end{abstract}

\section{INTRODUCTION}

Let $X$ be a compact manifold whose boundary $\partial X$ is the total space of a locally trivial fibration $\varphi: \partial X \rightarrow Y$ of closed manifolds. In contrast to the case of closed manifolds, a great number of algebras of pseudodifferential operators can naturally be associated to this geometric situation. To list a few of them, recall that Richard Melrose introduced a concept of geometric micro-localization that associates to certain classes of Lie algebras $\mathcal{V}$ of vector fields on $X$ algebras of pseudodifferential operators; the Lie algebra $\mathcal{V}$ is then also called a boundary fibration structure [26], [30]. The corresponding pseudodifferential calculus $\Psi_{\mathcal{V}}^{*}(X)$ contains the universal enveloping algebra $\operatorname{Diff}_{\mathcal{V}}^{*}(X)$ of $\mathcal{V}$, the $\mathcal{V}$-differential operators, as a subalgebra, and $\mathcal{V}$-elliptic (pseudo)differential operators (a notion that has a natural meaning in the context of boundary fibration structures) can be inverted within $\Psi_{\mathcal{V}}^{*}(X)$ up to operators of order $-\infty$. Possible candidates for boundary fibration structures on our manifold $X$ are, for instance,

$$
\begin{array}{rlrl}
\mathcal{V}_{e}(X):= & \left\{V \in \mathcal{C}^{\infty}(X, T X): V\right. \text { is } & \\
& \text { tangent to the fibers of } \pi \text { at } \partial X\} & & \text { (edge-structure) } \\
\mathcal{V}_{d e}(X):=\varrho_{N} \mathcal{V}_{e}(X) & & \text { (double-edge structure) } \\
\mathcal{V}_{\ell e}(X):=\varrho_{N}^{\ell} \mathcal{V}_{e}(X) & & \text { ( }(\text {-fold-edge structure), } \\
\mathcal{V}_{\Phi}(X):=\left\{V \in \mathcal{V}_{e}(X): V \varrho_{N} \in \varrho_{N}^{2} \mathcal{C}^{\infty}(X)\right\} & & \text { (fibered-cusp structure), }
\end{array}
$$

where $\varrho_{N}: X \rightarrow \overline{\mathbb{R}}_{+}$is a defining function for $\partial X$, i.e., $\partial X=\left\{\varrho_{N}=0\right\}$ and $d \varrho_{N}$ does not vanish on $\partial X$. Note that the fibered cusp structure $\mathcal{V}_{\Phi}(X)$ depends slightly on the choice of the boundary defining function. This dependence is discussed in

Received by the editors July 15, 2002 and, in revised form, January 16, 2003.

2000 Mathematics Subject Classification. Primary 58J42, 58J20.

Moroianu was partially supported by a DFG-grant (436-RUM 17/7/01) and by the European Commission RTN HPRN-CT-1999-00118 Geometric Analysis. 
more detail in 23]. Corresponding pseudodifferential calculi were constructed and studied in [22] (edge-structure), [17], 32] (double-edge structure), and [23, [31] (fibered-cusp structure). A pseudodifferential calculus for the $\ell$-fold edge structure has been considered so far only for the very special case $\pi=\mathrm{id}: \partial X \rightarrow Y$ and $\ell=2$, under the name quadratic scattering structure [46]. The reader can easily invent more Lie algebras that might introduce boundary fibration structures, but one should be warned that it is not at all straightforward (and sometimes even impossible) to establish the additional properties that ensure the existence of a pseudodifferential calculus. For more details, we refer to [26, 32] and the forthcoming book 25.

It is worth noting that by integrating appropriate Lie algebroids as Nistor did in [39] and using general groupoid techniques, (slightly smaller) pseudodifferential calculi associated to many interesting boundary fibration structures can be constructed simultaneously. We refer to the survey [18] for many examples of this construction.

To give an idea of how the different boundary fibration structures in (11) look locally, we use coordinates $(x, y, z) \in \overline{\mathbb{R}}_{+} \times \mathbb{R}_{y}^{n} \times \mathbb{R}_{z}^{m}$ in a local product decomposition near the boundary, where $x$ is the restriction of the boundary defining function $\varrho_{N}$, $y$ is a set of variables on the base $Y$ lifted through $\varphi$, and $z$ are variables in the fiber. Then any vector field in one of the boundary fibration structures from (1) can be written locally as a linear combination over $C^{\infty}(X)$ of the following basic vector fields:

$$
\begin{array}{llll}
x \partial_{x}, & x \partial_{y}, & \partial_{z} & \text { (edge-structure) } \\
x^{2} \partial_{x}, & x^{2} \partial_{y}, & x \partial_{z} & \text { (double-edge structure) } \\
x^{\ell+1} \partial_{x}, & x^{\ell+1} \partial_{y}, & x^{\ell} \partial_{z} & \text { ( } \text {-fold-edge structure) } \\
x^{2} \partial_{x}, & x \partial_{y}, & \partial_{z} & \text { (fibered-cusp structure) }
\end{array}
$$

In the present paper we compute the Hochschild homology and study its relation to index problems for the fibered cusp calculus, i.e., the pseudodifferential calculus associated to the fibered cusp structure. Starting with the work of Wodzicki [43, [4], [45] and Brylinski and Getzler [3], 4] on Hochschild and cyclic homology of (pseudo)differential operators and on non-commutative residues and Euler classes, homology of pseudodifferential operators has attracted much attention, not only because of its relation to index problems as explained for instance in [34] or [15], [16, but also because of its connections to the non-commutative geometry of Alain Connes [6]. Indeed, algebras of pseudodifferential operators are non-commutative algebras naturally associated to (singular) geometric situations (boundary fibration structures), and there are good reasons to expect that the study of invariants of these non-commutative algebras will reveal information about the geometry and help us to understand what a non-commutative manifold (possibly with singularities) is supposed to be [7].

The interest in the fibered cusp calculus originally introduced in [23, 31] has grown considerably since Nye and Singer [38] used this pseudodifferential calculus to prove an $L^{2}$-index theorem for Dirac operators on $S^{1} \times \mathbb{R}^{3}$, and Vaillant [42] applied the fibered cusp calculus to study spectral and index theory for Dirac operators on manifolds with generalized fibered cusps that occur for instance when compactifying rank one locally symmetric spaces [36].

From (2), we see that the fibered cusp structure interpolates between two interesting boundary fibration structures on manifolds with boundary, namely the 
scattering structure corresponding to the trivial fibration $\varphi=\mathrm{id}: \partial X \rightarrow Y=\partial X$, locally given by $x^{2} \partial_{x}$ and $x \partial_{y}$, and the cusp structure corresponding to $\varphi: \partial X \rightarrow$ $Y=\{\mathrm{pt}\}$ locally generated by $x^{2} \partial_{x}$ and $\partial_{y}$. For our purposes it is interesting to note that the scattering structure is also a special case of the double-edge structure with $\varphi: \partial X \rightarrow Y=\{\mathrm{pt}\}$. The Hochschild homology for various algebras associated to the cusp algebra has been computed in 34, whereas the Hochschild homology for the analogous algebras in the scattering setting can be extracted as special cases from the homology of the double-edge calculus in [14. We do not know any way to combine these results to get the Hochschild homology for the fibered cusp calculus directly; nevertheless, the results mentioned above can be used to double-check the computations of this paper. On the other hand, when comparing the results for the double-edge calculus with those for the fibered cusp calculus, we see that Hochschild homology groups yield a functional-analytic way of distinguishing between the two calculi.

The fibered cusp calculus

$$
\Psi_{\Phi}^{\mathbb{Z}, \mathbb{Z}}(X)=\bigcup_{k, m \in \mathbb{Z}} \Psi_{\Phi}^{m, k}(X)
$$

is naturally a bifiltered algebra, with the first filtration $(m)$ given by the symbolic order of the pseudodifferential operator whereas the second filtration $(k)$ corresponds to the (negative of the) order of vanishing at the boundary, more precisely at the $\Phi$-front face, a notion that is explained in Section [2]

The algebra $\Psi_{\Phi}^{\mathbb{Z}, \mathbb{Z}}(X)$ has three interesting ideals, namely

$$
\begin{aligned}
\Psi_{\Phi}^{-\infty, \mathbb{Z}}(X) & :=\bigcup_{k \in \mathbb{Z}} \bigcap_{m \in \mathbb{Z}} \Psi_{\Phi}^{m, k}(X), \\
\Psi_{\Phi}^{\mathbb{Z},-\infty}(X) & :=\bigcup_{m \in \mathbb{Z}} \bigcap_{k \in \mathbb{Z}} \Psi_{\Phi}^{m, k}(X), \\
\Psi_{\Phi}^{-\infty,-\infty}(X) & :=\bigcap_{k \in \mathbb{Z}} \bigcap_{m \in \mathbb{Z}} \Psi_{\Phi}^{m, k}(X) .
\end{aligned}
$$

The residual ideal $\Psi_{\Phi}^{-\infty,-\infty}$ corresponds to operators with Schwartz kernels in the space of all smooth half-densities that vanish to infinite order at the boundary; it is easily seen to have Hochschild homology only in dimension 0, where it is onedimensional, the isomorphism to $\mathbb{C}$ being given by the usual operator trace [34]. We are not going to stress this ideal any further. Following [34], let us denote the following quotients by

$$
\begin{aligned}
{ }^{\Phi} \mathcal{I}_{\partial} & :=\Psi_{\Phi}^{-\infty, \mathbb{Z}} / \Psi_{\Phi}^{-\infty,-\infty}, \\
{ }^{\Phi} \mathcal{A}_{\partial} & :=\Psi_{\Phi}^{\mathbb{Z}, \mathbb{Z}} / \Psi_{\Phi}^{\mathbb{Z},-\infty}, \\
{ }^{\Phi} \mathcal{I}_{\sigma} & :=\Psi_{\Phi}^{\mathbb{Z},-\infty} / \Psi_{\Phi}^{-\infty,-\infty} \\
{ }^{\Phi} \mathcal{A}_{\sigma} & :=\Psi_{\Phi}^{\mathbb{Z}, \mathbb{Z}} / \Psi_{\Phi}^{-\infty, \mathbb{Z}} \\
{ }^{\Phi} \mathcal{A}_{\partial, \sigma} & :=\Psi_{\Phi}^{\mathbb{Z}, \mathbb{Z}}(X) /\left(\Psi_{\Phi}^{-\infty, \mathbb{Z}}+\Psi_{\Phi}^{\mathbb{Z},-\infty}\right) .
\end{aligned}
$$


The following diagram summarizes the situation, where the horizontal and vertical sequences are exact:

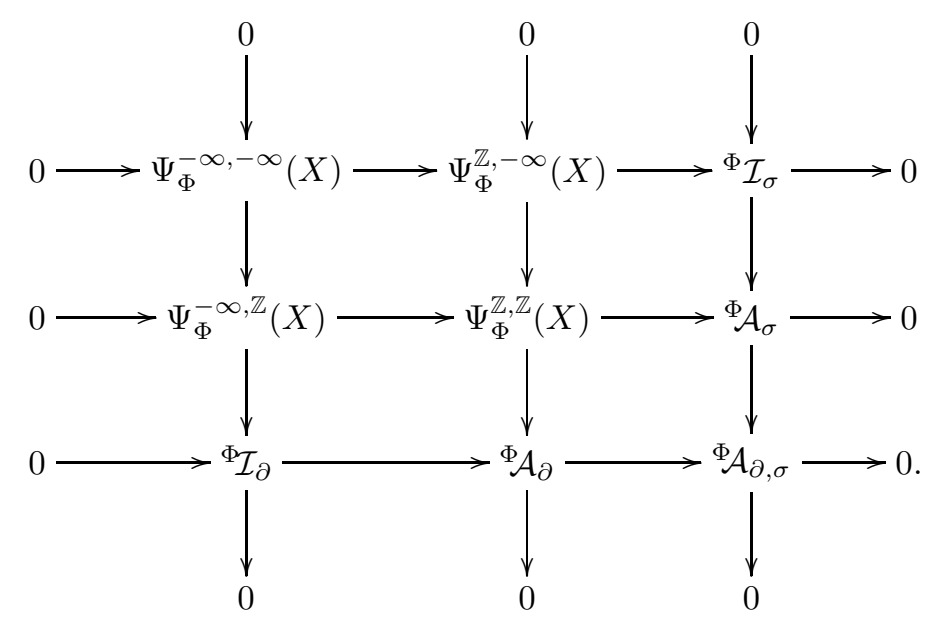

We are going to compute the Hochschild homology of the ideals ${ }^{\Phi} \mathcal{I}_{\sigma}$ and ${ }^{\Phi} \mathcal{I}_{\partial}$ as well as of the quotients ${ }^{\Phi} \mathcal{A}_{\sigma},{ }^{\Phi} \mathcal{A}_{\partial}$, and ${ }^{\Phi} \mathcal{A}_{\partial, \sigma}$. Moreover, we give geometric descriptions of the long exact sequences in Hochschild homology arising from the six short exact sequences (three rows and three columns) in (3). Not surprisingly, for the computation of the homology of the symbolic algebras ${ }^{\Phi} \mathcal{I}_{\sigma},{ }^{\Phi} \mathcal{A}_{\sigma}$, and ${ }^{\Phi} \mathcal{A}_{\partial, \sigma}$ we use the same approach as Brylinski and Getzler in [4, and obtain, for $k \in \mathbb{N}_{0}$ and with $N:=\operatorname{dim} X$,

$$
\begin{aligned}
H H_{k}\left({ }^{\Phi} \mathcal{I}_{\sigma}\right) & \cong H_{r e l}^{2 N-k}\left({ }^{\Phi} S^{*} X \times S^{1}\right), \\
H H_{k}\left({ }^{\Phi} \mathcal{A}_{\sigma}\right) & \cong H_{a b s}^{2 N-k}\left({ }^{\Phi} S^{*} X \times S^{1}\right) \oplus H^{2 N-1-k}\left(\left.{ }^{\Phi} S^{*} X\right|_{\partial X} \times S^{1}\right), \\
H H_{k}\left({ }^{\Phi} \mathcal{A}_{\partial, \sigma}\right) & \cong H^{2 N-k}\left(\left.{ }^{\Phi} S^{*} X\right|_{\partial X} \times S^{1} \times S^{1}\right),
\end{aligned}
$$

where $H_{r e l}^{*}$ (resp. $H_{a b s}^{*}$ ) stand for the relative (resp. absolute) de Rham cohomology of a compact manifold with boundary. The fibered cusp cosphere bundle ${ }^{\Phi} S^{*} X$ shows up naturally in the analysis of the fibered cusp calculus and is in fact diffeomorphic to the usual cosphere bundle $S^{*} X$, though not naturally. However, even though the idea of the computation is the same as in [4], namely to use an appropriate form of symplectic duality to identify the $d^{1}$ in the spectral sequence, convergence of the spectral sequence is an issue that is often neglected, and we spend some time to give the complete argument. In fact, we are naturally led to consider tri-filtered differential complexes, where the filtration indices run in $\mathbb{Z}$. In this setting convergence issues become very complicated, although it is rather satisfactory that they can be affirmatively answered. We give a general criterion for convergence which might well be applied in other situations.

More surprising is the computation of the homology of the boundary ideal ${ }^{\Phi} \mathcal{I}_{\partial}$ and the boundary algebra ${ }^{\Phi} \mathcal{A}_{\partial}$. We use a non-canonical morphism of algebras $\Theta: \Psi_{s c, c p t}^{-\infty}\left(Y_{\varepsilon}\right) \rightarrow \Psi_{\Phi}^{-\infty}(X)$ of the algebra of (compactly supported) scattering pseudodifferential operators of order $-\infty$ on the cylinder $Y_{\varepsilon}:=Y \times[0, \epsilon)$ to the smoothing fibered cusp operators on $X$ to reduce the computation of the Hochschild homology of ${ }^{{ }^{\mathcal{I}} \mathcal{I}_{\partial}}$ to that of the smoothing boundary ideal ${ }^{s} \mathcal{I}_{\partial}$ of the scattering 
calculus on $Y_{\varepsilon}$. More precisely, we have

$$
H H_{k}\left({ }^{\Phi} \mathcal{I}_{\partial}\right) \cong H^{n+1-k}(Y) \oplus H^{n-k}(Y)
$$

where, for simplicity, we have assumed that the base $Y$ of the fibration is orientable; the general case is obtained in Theorem 4.6 using cohomology with coefficients in the orientation bundle. The homology of the fibered cusp boundary algebra follows then from a long exact sequence that we describe explicitly in geometric terms (Proposition 6.1, Theorem 6.2).

Starting from a different approach using differentiable groupoids, Benameur and Nistor [2] developed a very general machinery that computes, when specializing to our setting, the $E^{\infty}$ term in the spectral sequence of the Hochschild homology of the symbolic algebra $\Phi_{\mathcal{A}}$. Finally, note that computing Hochschild homology for algebras of pseudodifferential operators is not restricted to the algebras mentioned above: for instance, the Hochschild homology for Boutet de Monvel's algebra on a compact manifold with boundary has been computed by Nest and Schrohe [37; the second author has computed the homology for the adiabatic limit algebra [35].

The second part of the paper is devoted to residue functionals, traces and index formulas for the fibered cusp calculus. As in [14] or [34, we introduce several linear functionals on $\Psi_{\Phi}^{\mathbb{Z}, \mathbb{Z}}(X)$ that descend to traces on some of the ideals and quotients considered above by taking appropriate coefficients in the Laurent expansion of the meromorphic extension $Z_{\varrho_{N}, Q}(A)$ of the double-zeta function $(\lambda, z) \mapsto \operatorname{Tr}\left(A \varrho_{N}^{z} Q^{-\lambda}\right)$; here, $Q \in \Psi_{\Phi}^{1,0}(X)$ is a fixed elliptic, symmetric, strictly positive operator. Using these functionals, we can give a formula for the index of Fredholm operators $A$ in the fibered cusp calculus that extends the Atiyah-PatodiSinger formula for closed manifolds in the form 34 to the fibered cusp calculus:

$$
\operatorname{index}(A)=\overline{A S}(A)-\lim _{a} \eta(A) / 2 .
$$

Here $\overline{A S}(A)$ is given in terms of Hochschild homological functionals that depend only on the symbolic behavior of the operator $A$ but still involve an inverse of $A$ up to trace class remainders, while $\lim _{a} \eta(A)$ is a term that depends only on the behavior of $A$ near the boundary. The term $\overline{A S}(A)$ can be linked to the asymptotics of the heat kernel [13. The notation $\lim _{a} \eta(A)$ is motivated by the fact that in the special case $Y=S^{1}$ and $A$ a differential operator with a Dirac-type decomposition near the boundary, the non-local boundary contribution to the index can be identified as the adiabatic limit of the eta invariant of the "restriction to the boundary" of $A$. This result is developed in [13].

The paper is organized as follows: In Section 2 we review some basic facts about the fibered cusp calculus; Section 3 is devoted to the construction of the morphism $\Theta: \Psi_{s c, c p t}^{-\infty}\left(Y_{\varepsilon}\right) \rightarrow \Psi_{\Phi}^{-\infty}(X)$, which is used in Section 4 to define a generalization of the Hochschild-Kostant-Rosenberg map in order to determine the Hochschild homology of $\Phi_{\mathcal{I}}$. The computation of the Hochschild homology of the symbol algebras ${ }^{\Phi} \mathcal{I}_{\sigma},{ }^{\Phi} \mathcal{A}_{\sigma}$, and ${ }^{\Phi} \mathcal{A}_{\partial, \sigma}$ can be found in Section 5. The long exact sequence in Hochschild homology arising from the boundary sequence

$$
0 \longrightarrow{ }^{\Phi} \mathcal{I}_{\partial} \longrightarrow{ }^{\Phi} \mathcal{A}_{\partial} \longrightarrow{ }^{\Phi} \mathcal{A}_{\partial, \sigma} \longrightarrow 0
$$

is described in Section [6] in geometric terms. In Section [7] we introduce and study various trace-like functionals on the fibered cusp calculus that generate the 0dimensional Hochschild homology groups. As in [16], [14], 34], these functionals 
can be used to derive an index formula for fully elliptic fibered cusp operators. The formula is presented in Section 8 .

The paper contains two appendices. In Appendix $\mathrm{A}$, for the convenience of the reader, we review the definition of Schwartz (resp. symbolic) sections of vector bundles. Appendix B contains the definition of the Hochschild chain spaces for topologically filtered algebras in the sense of [2], and a general criterion for the convergence of the spectral sequence associated to a tri-filtered complex, which is applied in the body of the paper to the Hochschild complexes of the symbol and boundary $\Phi$-algebras.

As usual, we write $S^{[m]}(V)$ for the space of all smooth functions $V \backslash\{0\} \rightarrow \mathbb{C}$ of a vector bundle $V$ that are positively homogeneous of degree $m \in \mathbb{C}$.

\section{ACKNOWLEDGMENTS}

We are indebted to Richard Melrose and Victor Nistor for explaining to us their results in [34], which greatly influenced this paper, and to an anonymous referee for a careful reading of the manuscript. The first named author would like to thank Michael Singer and Boris Vaillant for some private lessons on the $\Phi$-calculus.

\section{Review of the FIBERED CUSP CALCULUS}

In this section we review basic properties of the fibered cusp calculus. For details and most of the proofs we refer to [23], 31], and [42].

The fibered cusp structure space. Throughout this paper, $X=X^{N}$ stands for a smooth, compact manifold with boundary $\partial X$ that is the total space of a locally trivial fibration $\varphi: \partial X \rightarrow Y=Y^{n}$ of closed manifolds. For simplicity, let us assume that $\partial X$ is connected; so the fibers of $\varphi$ are all diffeomorphic, say to a closed manifold $F=F^{m}$. For the dimension $N$ of $X$ we thus have $N=n+m+1$. Furthermore, we fix a boundary defining function $\varrho_{N}: X \rightarrow \overline{\mathbb{R}}_{+}$for $\partial X$, i.e., $\varrho_{N}$ is a smooth function such that $\partial X=\left\{\varrho_{N}=0\right\}$ and $d \varrho_{N} \neq 0$ at $\partial X$.

Let $\mathcal{V}_{e}(X)$ be the Lie algebra of all edge vector fields, i.e., all smooth vector fields on $X$ that are tangent to the fibers of $\varphi$ at the boundary [22. Then the vector fields in

$$
\mathcal{V}_{\Phi}(X):=\left\{V \in \mathcal{V}_{e}(X): V \varrho_{N} \in \varrho_{N}^{2} \mathcal{C}^{\infty}(X)\right\}
$$

are called fibered cusp or simply $\Phi$-vector fields. Note that the definition of the Lie algebra $\mathcal{V}_{\Phi}(X)$ depends slightly on the choice of the boundary defining function $\varrho_{N}$ 23]. To give a local description of $\Phi$-vector fields near the boundary, let

$$
(x, y, z): X \supseteq U \longrightarrow \overline{\mathbb{R}}_{+} \times \mathbb{R}_{y}^{n} \times \mathbb{R}_{z}^{m}
$$

be coordinates of a local product decomposition near the boundary, i.e., $x=\left.\varrho_{N}\right|_{U}$, $y$ are variables on the base $Y$ lifted through $\varphi$, and with $z$ corresponding to coordinates on the fiber $F$. Then $V \in \mathcal{V}_{\Phi}(X)$ is of the form

$$
V(x, y, z)=a(x, y, z) x^{2} \partial_{x}+\sum_{j=1}^{n} b_{j}(x, y, z) x \partial_{y_{j}}+\sum_{k=1}^{m} c_{k}(x, y, z) \partial_{z_{k}}, \quad(x, y, z) \in U
$$

with coefficients $a, b_{j}, c_{k}$ smooth up to $x=0$. Thus, by the Serre-Swan theorem there exists a smooth vector bundle ${ }^{\Phi} T X \rightarrow X$ together with a map $\iota_{\Phi}:{ }^{\Phi} T X \rightarrow$ $T X$ of vector bundles over $X$ such that

$$
\iota_{\Phi}\left(\mathcal{C}^{\infty}\left(X,{ }^{\Phi} T X\right)\right)=\mathcal{V}_{\Phi}(X) .
$$


We call ${ }^{\Phi} T X$ the $\Phi$-tangent bundle, and its dual ${ }^{\Phi} T^{*} X$ the $\Phi$-cotangent bundle. Note that the compactness of $X$ is not necessary for the existence of the $\Phi$-tangent bundle ${ }^{\Phi} T X$. In the special case when the fibration $\varphi=$ id $: \partial X=Y \rightarrow Y$ is trivial, $\Phi$-vector fields are also known as scattering vector fields [28]; accordingly, in that case the $\Phi$-tangent bundle is called the scattering tangent bundle, and we write ${ }^{\Phi} T X={ }^{s c} T X$. By the very definition, the restriction of the canonical map $\iota_{\Phi}:{ }^{\Phi} T X \rightarrow T X$ and its dual $\iota_{\Phi}^{*}: T^{*} X \rightarrow{ }^{\Phi} T^{*} X$ to the interior $X_{0}:=X \backslash \partial X$ are isomorphisms. Thus, the canonical symplectic form $\omega$ on $\left.T^{*} X\right|_{X_{0}}$ can be pushed forward to a singular closed 2-form $\omega_{\Phi}$ on $\left.{ }^{\Phi} T^{*} X\right|_{X_{0}}$ giving $\left.{ }^{\Phi} T^{*} X\right|_{X_{0}}$ the structure of a symplectic manifold. With respect to local coordinates $(x, y, z)$ as in (4), and the associated local coordinates $(x, y, z, \xi, \eta, \zeta)$ on $\left.{ }^{\Phi} T^{*} X\right|_{U}, \omega_{\Phi}$ is given by

$$
\omega_{\Phi}=\frac{d x}{x^{2}} \wedge d \xi+\frac{d y}{x} \wedge d \eta+d z \wedge d \zeta+x \eta \frac{d x}{x^{2}} \wedge \frac{d y}{x} ;
$$

so $\omega_{\Phi} \in \mathcal{C}^{\infty}\left({ }^{\Phi} T^{*} X, \Lambda^{2}\left({ }^{\Phi} T^{*}\left({ }^{\Phi} T^{*} X\right)\right)\right)$. The associated $\Phi$-Poisson bracket $\{f, g\}_{\Phi}$ is locally given by

$$
\begin{aligned}
\{f, g\}_{\Phi}= & x^{2} \partial_{x} f \partial_{\xi} g-x^{2} \partial_{x} g \partial_{\xi} f+x \partial_{y} f \partial_{\eta} g-x \partial_{y} g \partial_{\eta} f \\
& +\partial_{z} f \partial_{\zeta} g-\partial_{z} g \partial_{\zeta} f+x\left(\eta \partial_{\eta} f \partial_{\xi} g-\eta \partial_{\eta} g \partial_{\xi} f\right)
\end{aligned}
$$

Let us denote the kernel of the map $\left.\iota_{\Phi}\right|_{\partial X}:\left.\left.{ }^{\Phi} T X\right|_{\partial X} \rightarrow T X\right|_{\partial X}$ by ${ }^{\Phi} N \partial X$. Note that ${ }^{\Phi} N \partial X$ is locally generated by the vector fields $x^{2} \partial_{x}$ and $x \partial_{y}$; hence, ${ }^{\Phi} N \partial X$ is a vector bundle over $\partial X$. Let $V \partial X \rightarrow \partial X$ be the vertical tangent bundle, i.e., the kernel of the differential $T \varphi: T \partial X \rightarrow T Y$. Then we have the following short exact sequence of vector bundles over $\partial X$ :

$$
0 \longrightarrow{ }^{\Phi} N \partial X \longrightarrow{ }^{\Phi} T X \longrightarrow V \partial X \longrightarrow 0 \text {. }
$$

In fact, the bundle ${ }^{\Phi} N \partial X$ is the pull-back $\varphi^{*}\left({ }^{\Phi} N Y\right)$ of a vector bundle ${ }^{\Phi} N Y$ over $Y$. Since this bundle plays an important role in our computations, let us recall one way of constructing it. For small $\varepsilon>0$, let $Y_{\varepsilon}:=Y \times[0, \varepsilon)$, and let ${ }^{s c} T Y_{\varepsilon}$ be the corresponding scattering tangent bundle. Then ${ }^{\Phi} N Y:=\left.{ }^{s c} T Y_{\varepsilon}\right|_{Y \times\{0\}}$ has the desired properties. Indeed, with respect to the local coordinates (4), the map $\vartheta:{ }^{\Phi} N \partial X \rightarrow{ }^{\Phi} N Y$ with $\vartheta\left(\left.x^{2} \partial_{x}\right|_{(0, y, z)}\right)=\left.x^{2} \partial_{x}\right|_{(y, 0)}$ and $\vartheta\left(\left.x \partial_{y}\right|_{(0, y, z)}\right)=\left.x \partial_{y}\right|_{(y, 0)}$ is well-defined, does not depend on the choice of local coordinates, and makes the following pull-back diagram commutative.

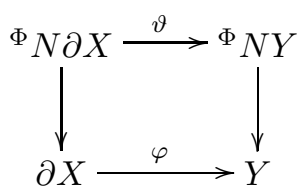

The fibered cusp double space. For the definition of the fibered cusp calculus we need a compact manifold with corners that is obtained by blowing up a sequence of $p$-submanifolds of the product $X^{2}$. For the concept of blowing up $p$-submanifolds we refer for instance to [10], 28 and the forthcoming book [25].

Let $\beta_{b}^{2}: X_{b}^{2}:=\left[X^{2} ;(\partial X)^{2}\right] \rightarrow X^{2}$ be the $b$-blow-up [27]. By [27, Lemma 4.1] the $b$-front face $\mathrm{ff}_{b}$, i.e., the new boundary face of $X_{b}^{2}$ obtained by the $b$-blow-up, is canonically diffeomorphic to the product $[-1,1] \times \partial X \times \partial X$. Note that we have fixed a boundary defining function $\varrho_{N}$ to define the fibered cusp structure. Then

$$
B_{\Phi}:=\left\{\left(0, q, q^{\prime}\right) \in \mathrm{ff}_{b}=[-1,1] \times \partial X \times \partial X: \varphi(q)=\varphi\left(q^{\prime}\right)\right\}
$$


is a $p$-submanifold of $X_{b}^{2}$, and

$$
\beta_{\Phi}^{2}: X_{\Phi}^{2}:=\left[X_{b}^{2} ; B_{\Phi}\right] \stackrel{\beta}{\longrightarrow} X_{b}^{2} \stackrel{\beta_{b}^{2}}{\longrightarrow} X^{2}
$$

is called the fibered cusp or briefly the $\Phi$-double space. It is a compact manifold with corners up to codimension two. Let $\Delta \subseteq X^{2}$ be the diagonal. Then the submanifold $\Delta_{\Phi}:=\overline{\left(\beta_{\Phi}^{2}\right)^{-1}\left(\Delta \backslash(\partial X)^{2}\right)} X_{\Phi}^{2}$ is said to be the lifted diagonal, and the boundary face $\mathrm{ff}_{\Phi}$ of $X_{\Phi}^{2}$ that meets the diagonal is called the fibered cusp or simply $\Phi$-front face. The $\Phi$-front face is of great importance for understanding the properties of the fibered cusp calculus near the boundary. As explained in [23, [42], $\mathrm{ff}_{\Phi}$ is canonically diffeomorphic to

$$
\mathrm{ff}_{\Phi} \cong \partial X \times_{Y}{ }^{\Phi} \bar{N} \partial X=\partial X \times_{Y} \partial X \times_{Y}{ }^{\Phi} \bar{N} Y,
$$

where ${ }^{\Phi} \bar{N} \partial X$ (resp. ${ }^{\Phi} \bar{N} Y$ ) is the radial compactification of ${ }^{\Phi} N \partial X$ (resp. ${ }^{\Phi} N Y$ ) as explained in Appendix A Consequently, the interior $\mathrm{ff}_{\Phi}^{\text {int }}$ of $\mathrm{ff}_{\Phi}$ is canonically diffeomorphic to $\partial X \times_{Y}{ }^{\Phi} N \partial X=\partial X \times_{Y} \partial X \times_{Y}{ }^{\Phi} N Y$. If necessary, we use local coordinates

$$
r=x+x^{\prime}, \quad S=\frac{x-x^{\prime}}{\left(x+x^{\prime}\right)^{2}}, \quad U=\frac{y-y^{\prime}}{x+x^{\prime}}, \quad y^{\prime}, z, z^{\prime}
$$

near $\mathrm{ff}_{\Phi}^{\text {int }}=\{r=0\}$. The lifted diagonal is then given by $\Delta_{\Phi}=\{S=0, U=0, z=$ $\left.z^{\prime}\right\}$. With respect to the decomposition (7) of the $\Phi$-front face $\mathrm{ff}_{\Phi}$, the variables $S \in \mathbb{R}_{S}$ and $U \in \mathbb{R}_{U}^{n}$ then correspond to the linear variables in the fibers of ${ }^{\Phi} N Y$.

The $\Phi$-density bundle. Finally, for a density bundle that is adapted to the fibered cusp calculus, apply the smooth functor $\Omega^{\alpha}$ of $\alpha$-densities to the $\Phi$-cotangent bundle ${ }^{\Phi} T^{*} X$ to obtain the bundle ${ }^{\Phi} \Omega^{\alpha}(X)$ of $\Phi$ - $\alpha$-densities. The choice of local product coordinates as in (44) trivializes $\left.{ }^{\Phi} \Omega^{\alpha}(X)\right|_{U}$. A non-vanishing section is given by $\left|\frac{d x}{x^{n+2}} d y d z\right|^{\alpha}$.

On the $\Phi$-double space $X_{\Phi}^{2}$, we use the $\Phi$-kernel-half density bundle $K D_{\Phi}^{1 / 2}:=$ $\varrho_{\mathrm{ff}_{\Phi}}^{-(2+n) / 2} \Omega^{1 / 2}\left(X_{\Phi}^{2}\right)$. It is completely characterized by the space of its $\mathcal{C}^{\infty}$-sections [27. Lemma 8.6], namely

$$
\mathcal{C}^{\infty}\left(X_{\Phi}^{2}, K D_{\Phi}^{1 / 2}\right)=\varrho_{\mathrm{ff}}^{-(2+n) / 2} \mathcal{C}^{\infty}\left(X_{\Phi}^{2}, \Omega^{1 / 2}\left(X_{\Phi}^{2}\right)\right) .
$$

Here, as usual, $\varrho_{\mathrm{ff}_{\Phi}}: X_{\Phi}^{2} \rightarrow \overline{\mathbb{R}}_{+}$stands for a defining function of the $\Phi$-front face $\mathrm{ff}_{\Phi}$.

The fibered cusp calculus. The fibered cusp calculus is defined by characterizing the singularities of the Schwartz kernel associated to a bounded linear operator $A: \dot{\mathcal{C}}^{\infty}\left(X,{ }^{\Phi} \Omega^{1 / 2}\right) \rightarrow \mathcal{C}^{-\infty}\left(X,{ }^{\Phi} \Omega^{1 / 2}\right)$. A convenient description of the Schwartz kernel $k_{A} \in \mathcal{C}^{-\infty}\left(X^{2},{ }^{\Phi} \Omega^{1 / 2} \otimes{ }^{\Phi} \Omega^{1 / 2}\right)$ is possible when replacing the double $X^{2}$ with the $\Phi$-double space $X_{\Phi}^{2}$. Recall that the blow-down map $\beta_{\Phi}^{2}: X_{\Phi}^{2} \rightarrow X^{2}$ induces via pull-back and duality an isomorphism

$$
\left(\beta_{\Phi}^{2}\right)_{*}: \mathcal{C}^{-\infty}\left(X_{\Phi}^{2}, K D_{\Phi}^{1 / 2}\right) \rightarrow \mathcal{C}^{-\infty}\left(X^{2},{ }^{\Phi} \Omega^{1 / 2} \otimes^{\Phi} \Omega^{1 / 2}\right) .
$$

We call the image $\kappa_{A}$ of $k_{A}$ under this isomorphism the lifted Schwartz kernel of A.

Definition 2.1. The space $\Psi_{\Phi}^{m}(X)$ of fibered cusp or briefly $\Phi$-operators of order $m \in \mathbb{C}$ consists of all continuous linear operators

$$
A: \dot{\mathcal{C}}^{\infty}\left(X,{ }^{\Phi} \Omega^{1 / 2}\right) \rightarrow \mathcal{C}^{-\infty}\left(X,{ }^{\Phi} \Omega^{1 / 2}\right)
$$


whose lifted Schwartz kernel $\kappa_{A}$ belongs to the space $I_{c l}^{m}\left(X_{\Phi}^{2}, \Delta_{\Phi} ; K D_{\Phi}^{1 / 2}\right)$ of classically conormal distributions that vanish to infinite order at all boundary faces of $X_{\Phi}^{2}$ other than the front face $\mathrm{ff}_{\Phi}$, and are extendible across $\mathrm{ff}_{\Phi}$.

A fibered cusp operator $A \in \Psi_{\Phi}^{m}(X)$ maps $\dot{\mathcal{C}}^{\infty}\left(X,{ }^{\Phi} \Omega^{1 / 2}\right)$ continuously into $\dot{\mathcal{C}}^{\infty}\left(X,{ }^{\Phi} \Omega^{1 / 2}\right)$; thus, composition of operators as well as conjugation with arbitrary complex powers of the boundary defining function $\varrho_{N}$ are well-defined. Since we get the lifted Schwartz kernel of $\varrho_{N}^{z} A \varrho_{N}^{-z}$ by multiplying the lifted Schwartz kernel of $A \in \Psi_{\Phi}^{m}(X)$ by a smooth function on the interior of $X_{\Phi}^{2}$ that extends smoothly to the front face, is identical to 1 at $\Delta_{\Phi}$ and polynomially bounded near all boundary faces other than the front face, we have $\varrho_{N}^{z} \Psi_{\Phi}^{m}(X) \varrho_{N}^{-z}=\Psi_{\Phi}^{m}(X)$, and we can define for $k \in \mathbb{C}$

$$
\Psi_{\Phi}^{m, k}(X):=\varrho_{N}^{-k} \Psi_{\Phi}^{m}(X)=\Psi_{\Phi}^{m}(X) \varrho_{N}^{-k} .
$$

Composition of operators leads to a bilinear map

$$
\Psi_{\Phi}^{m, k}(X) \times \Psi_{\Phi}^{m^{\prime}, k^{\prime}}(X) \rightarrow \Psi_{\Phi}^{m+m^{\prime}, k+k^{\prime}}(X)
$$

that defines on $\Psi_{\Phi}^{\mathbb{Z}, \mathbb{Z}}(X):=\bigcup_{m \in \mathbb{Z}} \bigcup_{k \in \mathbb{Z}} \Psi_{\Phi}^{m, k}(X)$ the bi-filtered algebra structure already considered in the introduction; in the sequel we use the notation introduced therein. We emphasize the negative sign in the definition of the second filtration, whose purpose is to make both filtrations increasing.

The filtration by the order of vanishing at the boundary on the algebra $\Psi_{\Phi}^{\mathbb{Z}, \mathbb{Z}}(X)$ induces in particular filtrations on ${ }^{\Phi} \mathcal{I}_{\partial}$ and ${ }^{\Phi} \mathcal{A}_{\partial}$, namely

$$
\begin{aligned}
\Phi_{\mathcal{I}}^{k} & :=\Psi_{\Phi}^{-\infty, k}(X) / \Psi_{\Phi}^{-\infty,-\infty}(X), \\
\Phi_{\mathcal{A}}^{m, k} & :=\Psi_{\Phi}^{m, k}(X) / \Psi_{\Phi}^{m,-\infty}(X)
\end{aligned}
$$

with quotients

$$
\begin{aligned}
& { }^{\Phi} \mathcal{I}_{\partial}^{[m]}:={ }^{\Phi} \mathcal{I}_{\partial}^{m} /{ }^{\Phi} \mathcal{I}_{\partial}^{m-1}=\Psi_{\Phi}^{-\infty, m}(X) / \Psi_{\Phi}^{-\infty, m-1}(X), \\
& \Phi^{\Phi} \mathcal{A}_{\partial}^{m,[k]}:={ }^{\Phi} \mathcal{A}_{\partial}^{m, k} /{ }^{\Phi} \mathcal{A}_{\partial}^{m, k-1}=\Psi_{\Phi}^{m, k}(X) / \Psi_{\Phi}^{m, k-1}(X) .
\end{aligned}
$$

As above, we let ${ }^{\Phi} \mathcal{I}_{\partial}^{[\mathbb{Z}]}:=\bigoplus_{m \in \mathbb{Z}}{ }^{\Phi} \mathcal{I}_{\partial}^{[m]}$, and so on. Similarly, the filtration of $\Psi_{\Phi}^{\mathbb{Z}, \mathbb{Z}}(X)$ by the operator order induces filtrations of ${ }^{\Phi} \mathcal{I}_{\sigma}$ and ${ }^{\Phi} \mathcal{A}_{\sigma}$, and we use the corresponding notation without any further comments. The usual symbol map for conormal distributions together with the fact that the density bundle of ${ }^{\Phi} T^{*} X$ is canonically trivialized by the symplectic form leads to the principal symbol map for the fibered cusp calculus,

$$
0 \longrightarrow \Psi_{\Phi}^{m-1, k}(X) \longrightarrow \Psi_{\Phi}^{m, k}(X) \stackrel{\Phi}{\sigma^{(m, k)}} S^{[m]}\left({ }^{\Phi} T^{*} X\right) \longrightarrow 0
$$

which is multiplicative in the obvious sense. Let ${ }^{\Phi} \bar{T}^{*} X$ be the radial compactification of the $\Phi$-cotangent bundle ${ }^{\Phi} T^{*} X$ in the sense of Appendix $\mathrm{A}$. Then the choice of a defining function

$$
\varrho_{\sigma}:{ }^{\Phi} \bar{T}^{*} X \rightarrow \overline{\mathbb{R}}_{+}
$$

for the $\Phi$-cosphere bundle

$$
{ }^{\Phi} S^{*} X:=\left({ }^{\Phi} T^{*} X \backslash\{0\}\right) / \mathbb{R}_{+} \subseteq{ }^{\Phi} \bar{T}^{*} X
$$

yields an identification of the space $S^{[m]}\left({ }^{\Phi} T^{*} X\right)$ with the space $\mathcal{C}^{\infty}\left({ }^{\Phi} S^{*} X\right)$. 
The normal homomorphism. In addition to the symbolic behavior at the lifted diagonal, the behavior of the lifted Schwartz kernel $\kappa_{A}$ of an operator $A \in \Psi_{\Phi}^{m}(X)$ at the $\Phi$-front face determines for instance the Fredholm properties of a fibered cusp operator. So, let us consider $\mathcal{N}_{\Phi}(A):=\left.\kappa_{A}\right|_{\mathrm{ff}_{\Phi}}$ directly. As shown in [23], the distributional density $\mathcal{N}_{\Phi}(A)$ can be understood as a family $\mathcal{N}_{\Phi}(A)(y), y \in Y$, of pseudodifferential operators of order $m$ on the space $\varphi^{-1}(y) \times{ }^{\Phi} N_{y} Y$ that are translation invariant with respect to the linear variables in ${ }^{\Phi} N_{y} Y$, and depend smoothly on $y \in Y$. The space of all these operators is denoted by $\Psi_{\operatorname{sus}\left({ }^{\Phi} N Y\right)-\varphi}^{m}(\partial X)$. For a precise definition we refer to 23]. The family $\mathcal{N}_{\Phi}(A)$ is called the normal operator of $A$, and $\mathcal{N}_{\Phi}$ induces a family of short exact sequences

$$
0 \longrightarrow \varrho_{N} \Psi_{\Phi}^{m}(X) \longrightarrow \Psi_{\Phi}^{m}(X) \stackrel{\mathcal{N}_{\Phi}}{\longrightarrow} \Psi_{\text {sus }\left({ }^{\Phi} N Y\right)-\varphi}^{m}(\partial X) \longrightarrow 0
$$

which are multiplicative in the obvious way. The normal operator extends to $A \in$ $\Psi_{\Phi}^{m, k}(X)$ by defining $\mathcal{N}_{\Phi}^{(k)}(A):=\mathcal{N}_{\Phi}\left(\varrho_{N}^{k} A\right)=\mathcal{N}_{\Phi}\left(A \varrho_{N}^{k}\right) \in \Psi_{\text {sus }\left({ }^{\Phi} N Y\right)-\varphi}^{m}(\partial X)$.

Fourier transform along the fibers of ${ }^{\Phi} N Y$, i.e., with respect to the variables $S$ and $U$ in (8), transforms a family $P=p\left(y, z, D_{z}, D_{S}, D_{U}\right) \in \Psi_{\left.\operatorname{sus}^{(\Phi} N Y\right)-\varphi}^{m}(\partial X)$ into a family $\widetilde{P}=\widetilde{p}\left(y, z, D_{z}, \xi, \eta\right)$ of pseudodifferential operators on $\varphi^{-1}(y)$ that depend smoothly on $y \in Y$ and symbolically of order $m$ on the dual variables $(\xi, \eta) \in{ }^{\Phi} N_{y}^{*} Y$ to $(S, U) \in{ }^{\Phi} N_{y} Y$ [31]. Let us write $\widetilde{\Psi}_{\operatorname{sus}\left({ }^{\Phi} N^{*} Y\right)-\varphi}^{m}(\partial X)$ for the corresponding space of operators, and $\widetilde{\mathcal{N}}_{\Phi}^{(k)}: \Psi_{\Phi}^{m, k}(X) \rightarrow \widetilde{\Psi}_{\operatorname{sus}\left(\Phi{ }^{*} Y\right)-\varphi}^{m}(\partial X)$ for the corresponding normal operator. For the sake of completeness, let us give a description of families in $\widetilde{\Psi}_{\operatorname{sus}\left({ }^{\Phi} N^{*} Y\right)-\varphi}(\partial X)$ using local data. By a partition of unity in $Y$ we can assume that the family $\widetilde{P} \in \widetilde{\Psi}_{\operatorname{sus}\left(\Phi_{N^{*}} Y\right)-\varphi}(\partial X)$ is compactly supported in an open set $V$ such that $\varphi^{-1}(V)=V \times F$. Then we have, up to a density factor in the $y$-variable,

$$
\widetilde{P} \in \mathcal{C}_{c}^{\infty}\left(V, \Psi^{M}\left(F ; \mathbb{R}_{\xi} \times \mathbb{R}_{\eta}^{n}\right)\right),
$$

where $\Psi^{M}\left(F ; \mathbb{R}_{\xi} \times \mathbb{R}_{\eta}^{n}\right)$ denotes the space of parameter-dependent or suspended pseudodifferential operators of order $M$ on $F$ in the sense of [40] - see for instance also [9], [19, 21] or 29] for the one-dimensional case. For $M=-\infty$, (10) is equivalent to $\widetilde{P} \in \mathcal{C}_{c}^{\infty}(V) \widehat{\otimes}_{\pi} \mathrm{S}\left(\mathbb{R}_{\xi} \times \mathbb{R}_{\eta}^{n}\right) \widehat{\otimes}_{\pi} \Psi^{-\infty}(F)$. For arbitrary $M \in \mathbb{C}$ a partition of unity in $F$ identifies $\widetilde{P}$ (up to operators of order $-\infty$ ) with a finite sum of families of the form $\widetilde{p}\left(y, z, D_{z}, \xi, \eta\right)$ for some compactly supported classical symbols $\tilde{p} \in \mathcal{C}_{c}^{\infty}\left(V, S_{c l}^{M}\left(\mathbb{R}_{z}^{m} ; \mathbb{R}_{\zeta}^{m} \times \mathbb{R}_{\xi} \times \mathbb{R}_{\eta}^{n}\right)\right)$.

In the sequel we use these equivalent pictures of the normal operator simultaneously.

Fiberwise trace for smoothing suspended operators. There exists a canonical trace on $\widetilde{\Psi}_{\operatorname{sus}\left(\Phi^{*} Y\right)-\varphi}^{-\infty}(\partial X)$, which we review here. An extension of this trace to families in $\widetilde{\Psi}_{\text {sus }\left({ }^{\Phi} N^{*} Y\right)-\varphi}(\partial X)$ for arbitrary finite $m \in \mathbb{Z}$ is constructed in Section 7 . As explained above, elements of $\widetilde{\Psi}_{\operatorname{sus}\left({ }^{\Phi} N^{*} Y\right)-\varphi}^{-\infty}(\partial X)$ are simply smooth sections of the half-density bundle $\Omega^{1 / 2}\left(\partial X \times_{Y} \partial X \times_{Y}{ }^{\Phi} \overline{N^{*}} Y\right)$ that vanish to infinite order at the boundary. Thus, inverse Fourier transform along the fibers of ${ }^{\Phi} N^{*} Y$ transforms an element $\widetilde{h} \in \widetilde{\Psi}_{\operatorname{sus}\left({ }^{\Phi} N^{*} Y\right)-\varphi}^{-\infty}(\partial X)$ into a smooth section $h$ of

$$
\Omega^{1 / 2}\left(\partial X \times_{Y} \partial X \times_{Y}{ }^{\Phi} \bar{N} Y\right)=\Omega^{1 / 2}\left(\mathrm{ff}_{\Phi}\right)
$$


that vanishes to infinite order at the boundary, i.e., we have $h \in \Psi_{\operatorname{sus}\left({ }^{\Phi} N Y\right)-\varphi}^{-\infty}(\partial X)$. Here we have used the identification $\mathrm{ff}_{\Phi} \cong \partial X \times_{Y} \partial X \times_{Y} \Phi \bar{N} Y$ by (7). Recall that the blow-down map $\beta_{\Phi}^{2}: X_{\Phi}^{2} \rightarrow X^{2}$ together with the projection $X^{2} \rightarrow X$ onto either the left or the right factor identifies $\Delta_{\Phi} \cap \mathrm{ff}_{\Phi}$ with $\partial X$. On the other hand, $\Delta_{\Phi} \cap \mathrm{ff}_{\Phi}$ corresponds under the diffeomorphism (17) to

$$
D_{\Phi}:=\left\{(q, q, v) \in \partial X \times_{Y} \partial X \times_{Y} \Phi \bar{N} Y: \varphi(q)=y \text { and } v=0 \in{ }^{\Phi} N_{y} Y\right\},
$$

and we have canonical isomorphisms

$$
\begin{aligned}
\Omega^{1 / 2} & \left.\left(\partial X \times_{Y} \partial X \times_{Y}{ }^{\Phi} \bar{N} Y\right)\right|_{D_{\Phi}} \\
& \left.\cong \varphi^{*} \Omega^{1 / 2}(Y) \otimes \Omega_{\text {fiber }}^{1 / 2}(\partial X) \otimes \Omega_{\text {fiber }}^{1 / 2}(\partial X) \otimes \varphi^{*} \Omega_{\text {fiber }}^{1 / 2}\left({ }^{\Phi} N Y\right)\right|_{Y} \\
& \cong \varphi^{*} \Omega^{1 / 2}(Y) \otimes \Omega_{\text {fiber }}^{1}(\partial X) \otimes \varphi^{*} \Omega^{1 / 2}(Y) \\
& \cong \Omega^{1}(\partial X),
\end{aligned}
$$

where (11) follows from the fact that the normal direction to the boundary in ${ }^{\Phi} N Y=\left.{ }^{s c} T Y_{\varepsilon}\right|_{Y \times\{0\}}$ leads to a canonical trivial factor in the fiber half-density bundle $\left.\Omega_{\text {fiber }}^{1 / 2}\left({ }^{\Phi} N Y\right)\right|_{Y}$. Thus, we can integrate $\left.h\right|_{\partial X}$ over $\partial X=\Delta_{\Phi} \cap \mathrm{ff}_{\Phi}$ and obtain a map

$$
\overline{\operatorname{Tr}}: \widetilde{\Psi}_{\operatorname{sus}\left({ }^{\Phi} N^{*} Y\right)-\varphi}^{-\infty}(\partial X) \longrightarrow \mathbb{C}:\left.\widetilde{h} \longmapsto \int_{\partial X} h\right|_{\partial X}
$$

satisfying

$$
\overline{\operatorname{Tr}}\left(\widetilde{h}_{1} \widetilde{h}_{2}\right)=\overline{\operatorname{Tr}}\left(\widetilde{h}_{2} \widetilde{h}_{1}\right)
$$

for $\widetilde{h}_{1} \in \widetilde{\Psi}_{\operatorname{sus}\left({ }^{\Phi} N^{*} Y\right)-\varphi}^{-\infty}(\partial X)$ and $\widetilde{h}_{2} \in \widetilde{\Psi}_{\operatorname{sus}\left({ }^{\Phi} N^{*} Y\right)-\varphi}^{m}(\partial X)$. Indeed, by a partition of unity in $Y$ we can assume that the family $\widetilde{h} \in \widetilde{\Psi}_{\operatorname{sus}\left({ }^{\Phi} N^{*} Y\right)-\varphi}^{-\infty}(\partial X)$ of pseudodifferential operators is compactly supported in an open set $V \subseteq Y$ such that $\varphi^{-1}(V) \cong$ $V \times F$. Then we have, up to the density factors, $\left.\widetilde{h}\right|_{V} \in \mathcal{C}_{c}^{\infty}\left(V, \Psi^{-\infty}\left(F ; \mathbb{R}_{\xi} \times \mathbb{R}_{\eta}^{n}\right)\right)$ and $\overline{\operatorname{Tr}}(\widetilde{h})=\int_{V} \int_{\mathbb{R}_{\xi}} \int_{\mathbb{R}_{\eta}^{n}} \operatorname{Tr}(\widetilde{h}(y)(\xi, \eta)) d \eta d \xi d y$, where $\operatorname{Tr}$ stands for the usual operator trace on operators on the fiber $F$. In particular, we see that $\overline{\operatorname{Tr}}$ vanishes on commutators because Tr has this property. Moreover, by gluing the local pieces together we obtain the following form of $\overline{\mathrm{Tr}}$ :

$$
\overline{\operatorname{Tr}}: \widetilde{\Psi}_{\operatorname{sus}\left({ }^{\Phi} N^{*} Y\right)-\varphi}^{-\infty}(\partial X) \longrightarrow \mathbb{C}: \widetilde{h} \longmapsto \int_{Y} \overline{\operatorname{Tr}}_{(y)}\left(\left.\widetilde{h}\right|_{\varphi^{-1}(y)}\right),
$$

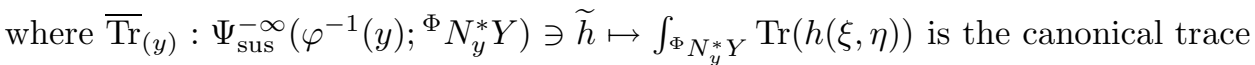
on the space of suspended operators on the fiber $\varphi^{-1}(y)$. For the one-suspended case the canonical trace $\overline{\operatorname{Tr}}$ was first considered in $[29$.

Moreover, by (12) or directly from the definition of $\overline{\mathrm{Tr}}$ we see that $\overline{\mathrm{Tr}}$ extends to $\widetilde{\Psi}_{\operatorname{sus}\left({ }^{\Phi} N^{*} Y\right)-\varphi}^{M}(\partial X)$ as long as $M<-N=-\operatorname{dim} X$.

Commuting fibered cusp operators with $\log \varrho_{N}$. Since $A \in \Psi_{\Phi}^{m, k}(X)$ as well as $\log \varrho_{N}$ act as bounded operators on $\dot{\mathcal{C}}^{\infty}\left(X,{ }^{\Phi} \Omega^{1 / 2}\right)$, we can consider their commutator $\left[A, \log \varrho_{N}\right]: \dot{\mathcal{C}}^{\infty}\left(X,{ }^{\Phi} \Omega^{1 / 2}\right) \rightarrow \dot{\mathcal{C}}^{\infty}\left(X,{ }^{\Phi} \Omega^{1 / 2}\right)$.

Lemma 2.2. The commutator with $\log \varrho_{N}$ yields a map

$$
\left[\cdot, \log \varrho_{N}\right]: \Psi_{\Phi}^{m, k}(X) \longrightarrow \Psi_{\Phi}^{m-1, k-1}(X) .
$$


Proof. Multiplication with $\log \varrho_{N}$ is a local operator; hence we can restrict ourselves to operators $A$ with lifted Schwartz kernel $\kappa_{A}$ supported in a coordinate patch of $X_{\Phi}^{2}$. Let us first assume that this patch is close to the intersection of the lifted diagonal $\Delta_{\Phi}$ with the fibered-cusp front face $\mathrm{ff}_{\Phi}$. We use local coordinates $\left(r, S, U, y^{\prime} z, z^{\prime}\right)$ as in (8). A straightforward computation shows that the lifted kernel $\kappa_{B}$ of $B:=\left[A, \log \varrho_{N}\right]$ is given by $\kappa_{B}=-\log \left(\frac{1+r S}{1-r S}\right) \kappa_{A}$. Since $\log \left(\frac{1+r S}{1-r S}\right)$ vanishes to first order at $\Delta_{\Phi}=\left\{S=0, U=0, z=z^{\prime}\right\}$ and $\mathrm{ff}_{\Phi}=\{r=0\}$ and is polynomially bounded for $|S| \rightarrow \infty$, we obtain $B \in \Psi_{\Phi}^{m-1, k-1}(X)$. The remaining cases are similar, only simpler.

The scattering calculus. As a rule, whenever the fibration $\varphi: \partial X \rightarrow Y$ is the identity map, i.e., whenever the fiber of $\varphi$ is a point, we replace the identifier $\Phi$ with $s c$ and talk about scattering instead of fibered cusp.

\section{SCATtering AND $\Phi$-OPERATORS OF ORDER $-\infty$}

We are going to show that for small $\varepsilon>0$ there exists a non-natural injective algebra homomorphism from smoothing scattering operators on $Y_{\varepsilon}$ to the algebra of smoothing fibered cusp-operators. This morphism $\Theta$ will be used in Section 4 to compute the Hochschild homology of the smoothing boundary ideal ${ }^{\Phi} \mathcal{I}_{\partial}$.

The choice of a normal fibration near the boundary $\partial X$ together with the boundary defining function $\varrho_{N}$ yields an open neighborhood $U_{\varepsilon}$ of the boundary and a diffeomorphism $U_{\varepsilon} \rightarrow \partial X \times[0, \varepsilon)$ for some $\varepsilon>0$ such that $\left.\varrho_{N}\right|_{U_{\varepsilon}}$ corresponds to the projection $\partial X \times[0, \varepsilon) \rightarrow[0, \varepsilon)$ onto the second factor. Moreover, $\left(U_{\varepsilon}\right)_{\Phi}^{2}:=\left(\beta_{\Phi}^{2}\right)^{-1}\left(U_{\varepsilon} \times U_{\varepsilon}\right) \subseteq X_{\Phi}^{2}$ is an open neighborhood of the front face $\mathrm{ff}_{\Phi}$, and for $k \in \mathbb{C}$ we can define

$$
\Psi_{\Phi, c p t}^{-\infty, k}\left(U_{\varepsilon}\right):=\varrho_{\mathrm{ff}_{\Phi}}^{-k}\left\{\kappa \in \mathcal{C}_{c}^{\infty}\left(\left(U_{\varepsilon}\right)_{\Phi}^{2}, K D_{\Phi}^{1 / 2}\left(\left(U_{\varepsilon}\right)_{\Phi}^{2}\right)\right): \kappa \equiv 0 \text { at } \partial\left(U_{\varepsilon}\right)_{\Phi}^{2} \backslash \mathrm{ff}_{\Phi}\right\},
$$

where $\varrho_{\mathrm{ff}_{\Phi}}:\left(U_{\varepsilon}\right)_{\Phi}^{2} \rightarrow \overline{\mathbb{R}}_{+}$is a defining function for the $\Phi$-front face. Because of the compact support condition, $\Psi_{\Phi, c p t}^{-\infty}\left(U_{\varepsilon}\right)$ is closed under composition, and the canonical inclusion $\Psi_{\Phi, c p t}^{-\infty}\left(U_{\varepsilon}\right) \hookrightarrow \Psi_{\Phi}^{-\infty}(X)$ is a morphism of algebras.

Let $Y_{\varepsilon}:=Y \times[0, \varepsilon)$. Then $U_{\varepsilon} \cong \partial X \times[0, \varepsilon) \stackrel{\varphi \times \text { id }}{\rightarrow} Y_{\varepsilon}$ is a locally trivial fiber bundle with fiber type $F$. As above, for $k \in \mathbb{C}$, let us define the compactly supported, smoothing scattering operators on $Y_{\varepsilon}$ by

$$
\Psi_{s c, c p t}^{-\infty, k}\left(Y_{\varepsilon}\right):=\varrho_{\mathrm{ff}_{s c}}^{-k}\left\{\kappa \in \mathcal{C}_{c}^{\infty}\left(\left(Y_{\varepsilon}\right)_{s c}^{2}, K D_{s c}^{1 / 2}\left(\left(Y_{\varepsilon}\right)_{s c}^{2}\right)\right): \kappa \equiv 0 \text { at } \partial\left(Y_{\varepsilon}\right)_{s c}^{2} \backslash \mathrm{ff}_{s c}\right\},
$$

where $\left(Y_{\varepsilon}\right)_{s c}^{2}$ is the scattering double space, $\mathrm{ff}_{s c}$ the scattering front face of $\left(Y_{\varepsilon}\right)_{s c}^{2}$, and $\varrho_{\mathrm{ff}_{s c}}:\left(Y_{\varepsilon}\right)_{s c}^{2} \rightarrow \overline{\mathbb{R}}_{+}$a defining function for $\mathrm{ff}_{s c}$. By the very definition, the fibration $U_{\varepsilon} \rightarrow Y_{\varepsilon}$ induces a fibration $\varphi_{\Phi}^{2}:\left(U_{\varepsilon}\right)_{\Phi}^{2} \rightarrow\left(Y_{\varepsilon}\right)_{s c}^{2}$ with fiber type $F \times F$.

Choose a fiber half-density $\nu \in \mathcal{C}^{\infty}\left(\partial X, \Omega_{\text {fiber }}^{1 / 2}\right)$ such that $\left.\int_{\varphi^{-1}(y)} \nu^{2}\right|_{\varphi^{-1}(y)}=1$ for all $y \in Y$, and let

$$
\begin{aligned}
\nu^{(2)}:=\nu \otimes \nu & \in \mathcal{C}^{\infty}\left(\partial X \times \partial X, \Omega_{\text {fiber }}^{1 / 2}(\partial X) \otimes \Omega_{\text {fiber }}^{1 / 2}(\partial X)\right) \\
& =\mathcal{C}^{\infty}\left(\partial X \times \partial X, \Omega_{\text {fiber }}^{1 / 2}(\partial X \times \partial X)\right) .
\end{aligned}
$$


We define

$$
\begin{aligned}
\Theta_{\varepsilon} & : \quad \Psi_{s c, c p t}^{-\infty, k}\left(Y_{\varepsilon}\right) \longrightarrow \Psi_{\Phi, c p t}^{-\infty, k}\left(U_{\varepsilon}\right): \kappa \longmapsto\left(\varphi_{\Phi}^{2}\right)^{*}(\kappa) \otimes \nu^{(2)} \\
\Theta & : \quad \Psi_{s c, c p t}^{-\infty, k}\left(Y_{\varepsilon}\right) \stackrel{\Theta_{\varepsilon}}{\longrightarrow} \Psi_{\Phi, c p t}^{-\infty, k}\left(U_{\varepsilon}\right) \hookrightarrow \Psi_{\Phi}^{-\infty, k}(X) .
\end{aligned}
$$

Before showing that the map $\Theta_{\varepsilon}$ is multiplicative, recall that the lifted Schwartz kernel $\kappa_{A B}$ of the composition of two fibered cusp operators $A$ and $B$ can be computed using a triple version $X_{\Phi}^{3}$ of the $\Phi$-double space $X_{\Phi}^{2}$. This space is a compact manifold with corners that comes equipped with three b-fibrations

$$
\pi_{O}^{\Phi}: X_{\Phi}^{3} \rightarrow X_{\Phi}^{2}, \quad O \in\{C, F, S\},
$$

where we have used the following projections:

$$
\begin{aligned}
& \pi_{C}: X^{3} \longrightarrow X^{2}:\left(q_{1}, q_{2}, q_{3}\right) \longmapsto\left(q_{1}, q_{3}\right), \\
& \pi_{F}: X^{3} \longrightarrow X^{2}:\left(q_{1}, q_{2}, q_{3}\right) \longmapsto\left(q_{2}, q_{3}\right), \\
& \pi_{S}: \quad X^{3} \longrightarrow X^{2}:\left(q_{1}, q_{2}, q_{3}\right) \longmapsto\left(q_{1}, q_{2}\right) .
\end{aligned}
$$

Using pull-back and push-forward under these b-fibrations, we obtain, up to a density factor,

$$
\kappa_{A B}=\left(\pi_{C}^{\Phi}\right)_{*}\left(\left(\pi_{S}^{\Phi}\right)^{*}\left(\kappa_{A}\right) \cdot\left(\pi_{F}^{\Phi}\right)^{*}\left(\kappa_{B}\right)\right) .
$$

The $\Phi$-triple space $X_{\Phi}^{3}$ has been constructed in [23], [42] in detail. Similarly, we can deal with the composition in the scattering calculus using the scattering triple space - for the triple space construction we refer to 28 .

Let $\left(U_{\varepsilon}\right)_{\Phi}^{3}$ (resp. $\left(Y_{\varepsilon}\right)_{s c}^{3}$ ) be the fibered cusp (resp. scattering) triple space of $U_{\varepsilon}$ (resp. $Y_{\varepsilon}$ ), and denote by $\pi_{O}^{\Phi}:\left(U_{\varepsilon}\right)_{\Phi}^{3} \rightarrow\left(U_{\varepsilon}\right)_{\Phi}^{2}$ (resp. $\pi_{O}^{s c}:\left(Y_{\varepsilon}\right)_{s c}^{3} \rightarrow\left(Y_{\varepsilon}\right)_{s c}^{2}$ ) the corresponding b-fibrations. Moreover, the fibration $U_{\varepsilon} \rightarrow Y_{\varepsilon}$ induces a fibration $\varphi_{\Phi}^{3}:\left(U_{\varepsilon}\right)_{\Phi}^{3} \rightarrow\left(Y_{\varepsilon}\right)_{s c}^{3}$ with fiber type $F^{3}$, and the following diagram is commutative:



Most important for us is the fact that the family of linear maps

$$
\Theta_{\varepsilon}=\Theta_{\varepsilon}^{(k)}: \Psi_{s c, c p t}^{-\infty, k}\left(Y_{\varepsilon}\right) \rightarrow \Psi_{\Phi, c p t}^{-\infty, k}\left(U_{\varepsilon}\right), \quad k \in \mathbb{C},
$$

is compatible with the composition of operators.

Theorem 3.1. Let $A \in \Psi_{s c, c p t}^{-\infty, k}\left(Y_{\varepsilon}\right)$ and $B \in \Psi_{s c, c p t}^{-\infty, \ell}\left(Y_{\varepsilon}\right)$ be arbitrary. Then we have

$$
\Theta_{\varepsilon}^{(k+\ell)}(A B)=\Theta_{\varepsilon}^{(k)}(A) \Theta_{\varepsilon}^{(\ell)}(B) \in \Psi_{\Phi, c p t}^{-\infty, k+\ell}\left(U_{\varepsilon}\right)
$$


Proof. It suffices to show that $\Theta_{\varepsilon}$ is multiplicative. We use the triple space construction and (15) for the kernel of the composition of two operators. Using the commutativity of (16), we obtain

$$
\begin{aligned}
\Theta_{\varepsilon}(A) \Theta_{\varepsilon}(B) & =\left[\left(\varphi_{\Phi}^{2}\right)^{*} \kappa_{A} \otimes \nu^{(2)}\right] \circ\left[\left(\varphi_{\Phi}^{2}\right)^{*} \kappa_{B} \otimes \nu^{(2)}\right] \\
& =\left(\pi_{C}^{\Phi}\right)_{*}\left(\left[\left(\pi_{S}^{\Phi}\right)^{*}\left(\left(\varphi_{\Phi}^{2}\right)^{*} \kappa_{A} \otimes \nu^{(2)}\right)\right] \cdot\left[\left(\pi_{F}^{\Phi}\right)^{*}\left(\left(\varphi_{\Phi}^{2}\right)^{*} \kappa_{B} \otimes \nu^{(2)}\right)\right]\right) \\
& =\left(\pi_{C}^{\Phi}\right)_{*}\left(\left[\left(\left(\varphi_{\Phi}^{3}\right)^{*}\left(\pi_{S}^{s c}\right)^{*} \kappa_{A}\right) \otimes \nu_{S}^{(2)}\right] \cdot\left[\left(\left(\varphi_{\Phi}^{3}\right)^{*}\left(\pi_{F}^{s c}\right)^{*} \kappa_{B}\right) \otimes \nu_{F}^{(2)}\right]\right) \\
& =\left(\varphi_{\Phi}^{2}\right)^{*}\left[\left(\pi_{C}^{s c}\right)_{*}\left(\left(\pi_{S}^{s c}\right)^{*} \kappa_{A} \cdot\left(\pi_{F}^{s c}\right)^{*} \kappa_{B}\right)\right] \otimes \nu^{(2)} \\
& =\left(\varphi_{\Phi}^{2}\right)^{*}\left(\kappa_{A B}\right) \otimes \nu^{(2)} \\
& =\Theta_{\varepsilon}(A B) .
\end{aligned}
$$

Recall the definitions

$$
\begin{aligned}
{ }^{s c} \mathcal{I}_{\partial} & =\Psi_{s c, c p t}^{-\infty, \mathbb{Z}}\left(Y_{\varepsilon}\right) / \Psi_{s c, c p t}^{-\infty,-\infty}\left(Y_{\varepsilon}\right), \\
{ }^{\Phi} \mathcal{I}_{\partial} & =\Psi_{\Phi}^{-\infty, \mathbb{Z}}(X) / \Psi_{\Phi}^{-\infty,-\infty}(X) .
\end{aligned}
$$

Note that the algebra ${ }^{s} \mathcal{I}_{\partial}$ does not depend on the particular choice of $\varepsilon>0$. By (14), the family of linear maps $\Theta=\Theta^{(k)}: \Psi_{s c, c p t}^{-\infty, k}\left(Y_{\varepsilon}\right) \rightarrow \Psi_{\Phi}^{-\infty, k}(X)$ then induces the desired linear map $\Theta:{ }^{s} \mathcal{I}_{\partial} \rightarrow{ }^{\Phi} \mathcal{I}_{\partial}$.

Corollary 3.2. The map $\Theta:{ }^{s} \mathcal{I}_{\partial} \rightarrow{ }^{\Phi} \mathcal{I}_{\partial}$ is a (non-natural) morphism of algebras.

\section{The generalized Hochschild-Kostant-Rosenberg maP AND THE HOMOLOGY OF THE SMOOTHING BOUNDARY IDEAL}

We refer to Appendix B for a review of topological Hochschild homology and for a summary of the notation that we are going to use in the sequel.

The algebra map $\Theta$ constructed in Section 3 induces a map of complexes with the same name between the Hochschild complexes

$$
\Theta: C_{*}\left({ }^{s} \mathcal{I}_{\partial}\right) \longrightarrow C_{*}\left({ }^{\Phi} \mathcal{I}_{\partial}\right)
$$

compatible with the boundary filtration. We will use this map to compare the two spectral sequences constructed with respect to this filtration. In fact, we get an isomorphism at ${ }^{\partial} E^{0}$; together with the convergence of the two spectral sequences, this implies that $\Theta$ induces an isomorphism on Hochschild homology.

The graded algebra ${ }^{s c} \mathcal{I}_{\partial}^{[\mathbb{Z}]}$ is naturally isomorphic to the commutative algebra of Laurent polynomials in $x$ with coefficients Schwartz functions on ${ }^{\Phi} N^{*} Y$. Thus, the Hochschild-Kostant-Rosenberg map HKR induces an isomorphism from the Hochschild homology of ${ }^{s c} \mathcal{I}_{\partial}^{[\mathbb{Z}]}$ to the space of forms (17). At the same time, $\Theta$ induces a map between the Hochschild complexes of ${ }^{s} \mathcal{I}_{\partial}^{[\mathbb{Z}]}$ and ${ }^{\Phi} \mathcal{I}_{\partial}^{[\mathbb{Z}]}$ :




Our plan is to construct a map $K: C_{k}\left({ }^{\Phi} \mathcal{I}_{\partial}^{[\mathbb{Z}]}\right) \rightarrow \bigoplus_{*} \Lambda_{\mathrm{S}}^{*}\left({ }^{\Phi} N^{*} Y\right) \otimes \Lambda^{k-*}\left(\mathbb{C}\left[x, x^{-1}\right]\right)$ making the diagram (17) commutative and inducing an injection of $H H_{*}\left({ }^{\Phi} \mathcal{I}_{\partial}^{[\mathbb{Z}]}\right)$ into $\bigoplus_{*} \Lambda_{\mathrm{S}}^{*}\left({ }^{\Phi} N^{*} Y\right) \otimes \Lambda^{k-*}\left(\mathbb{C}\left[x, x^{-1}\right]\right)$. The existence of $K$ with the above properties will show that $\Theta$ becomes an isomorphism on $H H\left({ }^{\Phi} \mathcal{I}_{\partial}^{[\mathbb{Z}]}\right)$. In this section we will only use $K$ on chains in $C_{k}\left({ }^{\Phi} \mathcal{I}_{\partial}^{[\mathbb{Z}]}\right)$, but in Section $[$ it will be crucial to apply $K$ on certain chains in the full graded boundary algebra ${ }^{\Phi} \mathcal{A}_{\partial}^{[\mathbb{Z}]}$; so we give here the general construction.

In order to define $K$ we must first construct a covariant derivative on ${ }^{\Phi} \mathcal{A}_{\partial}^{\mathbb{Z},[\mathbb{Z}]}$. Let us explain this notion. Consider the sheaf of rings $\operatorname{Pol}\left({ }^{\Phi} N^{*} Y \times \mathbb{R}\right)$ of polynomial functions on ${ }^{\Phi} N^{*} Y \times \mathbb{R}$. The algebra ${ }^{\Phi} \mathcal{A}_{\partial}^{\mathbb{Z},[\mathbb{Z}]}$ is a sheaf of $\operatorname{Pol}\left({ }^{\Phi} N^{*} Y \times \mathbb{R}\right)$-algebras over ${ }^{\Phi} N^{*} Y \times \mathbb{R}$, where the $\mathbb{R}$ factor corresponds to the variable $x$. Consider the sheaf $\mathcal{V}^{\text {Pol }}\left({ }^{\Phi} N^{*} Y \times \mathbb{R}\right)$ of vector fields with polynomial coefficients on ${ }^{\Phi} N^{*} Y \times \mathbb{R}$. A covariant derivative on ${ }^{\Phi} \mathcal{A}_{\partial}^{\mathbb{Z},[\mathbb{Z}]}$ is then defined as a map of sheaves

$$
\mathcal{V}^{\mathrm{Pol}}\left({ }^{\Phi} N^{*} Y \times \mathbb{R}\right) \otimes{ }^{\Phi} \mathcal{A}_{\partial}^{\mathbb{Z},[\mathbb{Z}]} \longrightarrow{ }^{\Phi} \mathcal{A}_{\partial}^{\mathbb{Z},[\mathbb{Z}]}
$$

that is $\operatorname{Pol}\left({ }^{\Phi} N^{*} Y \times \mathbb{R}\right)$-linear in the first argument and a derivation in the second. More precisely,

$$
\begin{aligned}
\nabla_{V}(A B) & =\left(\nabla_{V} A\right) B+A \nabla_{V} B \\
\nabla_{f V} A & =f \nabla_{V} A .
\end{aligned}
$$

Fix a connection $\nabla$ in the bundle $\Omega_{\text {fiber }}^{1 / 2}$ of fiberwise half-densities on $\partial X$ and a connection in the fibration $\varphi: \partial X \rightarrow Y$, that is, a rule for lifting vectors from $Y$ to $\partial X$. Pull back these connections through the maps $\partial X \times_{Y}{ }^{\Phi} N^{*} Y \rightarrow \partial X$ and ${ }^{\Phi} N^{*} Y \rightarrow Y$, respectively. They induce a connection in the fibration $\partial X \times_{Y}$ ${ }^{\Phi} N^{*} Y \rightarrow{ }^{\Phi} N^{*} Y$ and a covariant derivative on the pull-back of $\Omega_{\text {fiber }}^{1 / 2}$ to $\partial X \times_{Y}$ ${ }^{\Phi} N^{*} Y$. For a vector $V$ tangent to ${ }^{\Phi} N^{*} Y$, we denote by $\nabla_{V}$ the covariant derivative in the direction of the horizontal lift of $V$.

Lemma 4.1. The covariant derivative $\nabla$ maps the space

$$
\operatorname{Pol}\left({ }^{\Phi} N^{*} Y\right) \otimes \mathrm{S}\left(\partial X \times_{Y}{ }^{\Phi} N^{*} Y, \Omega_{\text {fiber }}^{1 / 2}\right)
$$

into the space

$$
\mathrm{S}\left(\partial X \times{ }_{Y}{ }^{\Phi} N^{*} Y, \Omega_{\text {fiber }}^{1 / 2}\right) .
$$

Proof. Clear in local coordinates in $Y$.

The elements of the algebra ${ }^{\Phi} \mathcal{A}_{\partial}^{\mathbb{Z},[0]}$ are fiberwise half-densities on $\mathrm{ff}_{\Phi}^{\text {int }}$. There are two ways of seeing $\mathrm{ff}_{\Phi}^{\text {int }} \cong \partial X \times_{Y} \partial X \times_{Y} \times{ }^{\Phi} N^{*} Y$ as a fibration over $\partial X \times_{Y}{ }^{\Phi} N^{*} Y$, corresponding to the two projections onto the left factor $\left(\pi_{L}\right)$ (resp. the right factor $\left.\left(\pi_{R}\right)\right)$. Thus, if $A \in{ }^{\Phi} \mathcal{A}_{\partial}^{\mathbb{Z},[0]}$ and $s \in \mathrm{S}\left(\partial X \times{ }_{Y}{ }^{\Phi} N^{*} Y, \Omega_{\text {fiber }}^{1 / 2}\right)$, let

$$
A s:=\left(\pi_{L}\right)_{*}\left(A \pi_{R}^{*} s\right) \in \mathrm{S}\left(\partial X \times_{Y}{ }^{\Phi} N^{*} Y, \Omega_{\text {fiber }}^{1 / 2}\right) .
$$

This defines a faithful action of ${ }^{\Phi} \mathcal{A}_{\partial}^{\mathbb{Z},[0]}$ on $\mathrm{S}\left(\partial X \times_{Y}{ }^{\Phi} N^{*} Y, \Omega_{\text {fiber }}^{1 / 2}\right)$; hence, for $V \in$ $\operatorname{Pol}^{\Phi} N^{*} Y$, we can define the action of $\nabla_{V}$ on ${ }^{\Phi} \mathcal{A}_{\partial}^{\mathbb{Z},[0]}$ by duality:

$$
\nabla_{V}(A)(s):=\nabla_{V}(A(s))-A\left(\nabla_{V}(s)\right) \in \mathrm{S}\left(\partial X \times_{Y}{ }^{\Phi} N^{*} Y, \Omega_{\text {fiber }}^{1 / 2}\right) .
$$

Finally, combining with the canonical action of $\partial / \partial x$ on $\mathbb{C}\left[x, x^{-1}\right]$, we get a covariant derivative on ${ }^{\Phi} \mathcal{A}_{\partial}^{\mathbb{Z},[0]} \otimes \mathbb{C}\left[x, x^{-1}\right]={ }^{\Phi} \mathcal{A}_{\partial}^{\mathbb{Z},[\mathbb{Z}]}$, as claimed. Properties (18) and (19) are 
easy to check. The covariant derivative preserves the ideal ${ }^{\Phi} \mathcal{I}_{\partial}^{\mathbb{Z},[\mathbb{Z}]}$. In the scattering case, the definition of $\nabla_{V}$ is independent of choices; so we write it simply as $V$. There exists a formula relating the algebra map $\Theta$ from Section 3 with $\nabla$.

Proposition 4.2. Let $A_{0}, B_{0} \in{ }^{s c} \mathcal{I}_{\partial}^{[0]} \otimes \mathbb{C}\left[x, x^{-1}\right]={ }^{s c} \mathcal{I}_{\partial}^{[\mathbb{Z}]}$. Then

$$
\nabla_{V}\left(\Theta\left(A_{0}\right)\right) \Theta\left(B_{0}\right)=\Theta\left(V\left(A_{0}\right) B_{0}\right) \text {. }
$$

Proof. A little care should be exercised, because the "naive" statement $\nabla_{V} \Theta(A)=$ $\Theta(V(A))$ does not hold. Essentially, the fiberwise density $\nu^{2}$ used to define $\Theta$ is not parallel, while its volume (which was chosen to be 1 ) is. We can clearly reduce ourselves to proving the statement for $A_{0}, B_{0} \in{ }^{s c} \mathcal{I}_{\partial}^{[0]}$. Note that ${ }^{s c} \mathcal{I}_{\partial}^{[0]}$ acts faithfully on $\mathrm{S}\left({ }^{\Phi} N^{*} Y\right.$ ) (by specializing from $\Phi$ to scattering the action described above). On the graded ideals, the map $\Theta$ can be described in terms of these actions by

$$
\Theta\left(A_{0}\right)(s)=\pi^{*}\left(A_{0}\left(\pi_{*}(s \otimes \nu)\right)\right) \otimes \nu,
$$

where $\pi$ is the projection $\partial X \times_{Y}{ }^{\Phi} N^{*} Y \rightarrow{ }^{\Phi} N^{*} Y$. Now $\pi_{*}\left(\pi^{*}\left(s_{0}\right) \otimes \nu^{2}\right)=s_{0}$ for all $s_{0} \in \mathrm{S}\left({ }^{\Phi} N^{*} Y\right)$, because $\nu^{2}$ has volume 1. Using this, the fact that $\pi_{*}\left(\nabla_{V}\left(\nu^{2}\right)\right)=$ $V\left(\pi_{*}\left(\nu^{2}\right)\right)=V(1)=0$, and the identity $\nabla_{V}\left(\pi^{*} s_{0}\right)=\pi^{*}\left(V\left(s_{0}\right)\right.$, valid for any connection, the rest of the proof is straightforward.

We can now define the map $K$. This is done as in 35. along the lines of a map constructed in [33]. Let $A=A_{0} \otimes \ldots \otimes A_{k}$ be a $k$-Hochschild chain in $C_{k}\left({ }^{\Phi} \mathcal{I}_{\partial}^{[\mathbb{Z}]}\right)$. For $V_{1}, \ldots, V_{k}$ polynomial vector fields (or derivations in $\mathbb{C}\left[x, x^{-1}\right]$ ), define

$$
K(A)\left(V_{1}, \ldots, V_{k}\right):=\sum_{\sigma \in \Sigma_{k}}(-1)^{|\sigma|} \overline{\operatorname{Tr}}\left(A_{0} \nabla_{V_{\sigma(1)}} A_{1} \ldots \nabla_{V_{\sigma(k)}} A_{k}\right) .
$$

Here $\overline{\operatorname{Tr}}$ denotes the fiberwise trace of smoothing suspended operators as explained in Section 2 .

Remark 4.3. It is clear from the definition that $K$ is still well-defined for tensor products $A=A_{0} \otimes \ldots \otimes A_{k}$ with $A_{j} \in \Psi_{\Phi}^{m_{j},[\mathbb{Z}]}(X)$ such that $m_{j} \in \mathbb{C}$ and

$$
\Re\left(m_{0}+\ldots+m_{k}\right)<-m=\operatorname{dim}(F),
$$

because the fiberwise trace still makes sense. In that case $K(A)$ is a form with symbol coefficients of order $\Re\left(m_{0}+\ldots+m_{k}\right)+m$.

We will use this fact later on.

Proposition 4.4. (1) $K \circ b=0$.

(2) $K \circ \Theta=H K R$.

(3) $K$ is injective on $H H\left({ }^{\Phi} \mathcal{I}_{\partial}^{[\mathbb{Z}]}\right)$.

Proof. The first two claims are straightforward, using Proposition 4.2. For the third, we use the strategy of [35, Proposition 5.4.9] to show that every cycle is homologous to a cycle that belongs to the image of $\Theta$. Then the first two assertions together with the Hochschild-Kostant-Rosenberg theorem yield the result.

The first two statements of Proposition 4.4 show that (17) commutes. From (17) it follows that $\Theta$ is injective on $H H\left({ }^{s c} \mathcal{I}_{\partial}^{[\mathbb{Z}]}\right)$, because $H K R$ is an isomorphism on homology. The third statement of Proposition 4.4 implies that $\Theta$ is also surjective from $H H\left({ }^{s c} \mathcal{I}_{\partial}^{[\mathbb{Z}]}\right)$ to $H H\left({ }^{\Phi} \mathcal{I}_{\partial}^{[\mathbb{Z}]}\right)$. Note now that these homology groups are just 
${ }^{\partial} E^{1}\left({ }^{s c} \mathcal{I}_{\partial}\right)$, respectively ${ }^{\partial} E^{1}\left({ }^{\Phi} \mathcal{I}_{\partial}\right)$. In conclusion $\Theta$ becomes an isomorphism at the level of ${ }^{\partial} E^{1}$ between the spectral sequences of ${ }^{s} \mathcal{I}_{\partial}$ and ${ }^{\Phi} \mathcal{I}_{\partial}$.

The homology of ${ }^{s c} \mathcal{I}_{\partial}$ is a particular case of [14, Theorem 4.15] in the double-edge case. So we have

\section{Proposition 4.5.}

$$
H H_{k}\left({ }^{s c} \mathcal{I}_{\partial}\right)=H_{\mathrm{S}}^{2 n+2-k}\left({ }^{\Phi} N^{*} Y\right) \oplus H_{\mathrm{S}}^{2 n+1-k}\left({ }^{\Phi} N^{*} Y\right) \frac{d x}{x} .
$$

However, the proof from [14 is quite inexplicit, since it makes use of a Čech complex adapted to Hochschild homology. We can give a better proof of this result in the scattering case.

Form the spectral sequence ${ }^{\partial} E$ with respect to the boundary filtration. We have just seen that

$$
{ }^{\partial} E_{i, j}^{1}\left({ }^{s c} \mathcal{I}_{\partial}\right)=\Lambda_{\mathrm{S}}^{i+j}\left({ }^{\Phi} N^{*} Y\right) x^{-i} \oplus \Lambda_{\mathrm{S}}^{i+j-1}\left({ }^{\Phi} N^{*} Y\right) x^{-1-i} d x .
$$

We claim that, up to sign,

$$
d^{1}=*_{s c} d *_{s c}
$$

where $d$ is the de Rham differential, and $*_{s c}:=\left(\imath_{s c}^{*}\right)^{-1} * \imath_{s c}^{*}$ is the conjugate of Brylinski's symplectic duality operator by the dual $\imath_{s c}^{*}$ of the scattering structure map $\imath_{s c}:{ }^{s c} T Y_{\varepsilon} \rightarrow T Y_{\varepsilon}$. Indeed, this follows as in [3] from the formula of the normal operator of a commutator: if $A \in{ }^{s} \mathcal{I}_{\partial}^{i}, B \in{ }^{s c} \mathcal{I}_{\partial}^{j}$, then $\mathcal{N}_{\Phi}^{[i+j-1]}([A, B])=$ $\left\{\mathcal{N}_{\Phi}^{[i]}(A), \mathcal{N}_{\Phi}^{[j]}(B)\right\}_{s c}$, where $\{,\}_{s c}:=\left(\imath_{s c}^{*}\right)^{-1}\{,\} \imath_{s c}^{*}$ is the scattering Poisson bracket.

We now note that the isomorphism $*_{s c}$ splits according to $x$-degree as follows:

$$
\begin{aligned}
*_{s c} & : \Lambda_{\mathrm{S}}^{k}\left({ }^{\Phi} N^{*} Y\right) x^{l} \oplus \Lambda_{\mathrm{S}}^{k-1}\left({ }^{\Phi} N^{*} Y\right) x^{l-1} d x \\
& \longrightarrow \Lambda_{\mathrm{S}}^{2 n+2-k}\left({ }^{\Phi} N^{*} Y\right) x^{l+k-n-1} \oplus \Lambda_{\mathrm{S}}^{2 n+1-k}\left({ }^{\Phi} N^{*} Y\right) x^{l+k-n-2} d x
\end{aligned}
$$

The homology of homogeneous forms in $x$ is concentrated in homogeneity 0 . Together with (21), it follows that

$$
{ }^{\partial} E_{i, j}^{2}\left({ }^{s} \mathcal{I}_{\partial}\right)= \begin{cases}H_{\mathrm{S}}^{n+1-i}\left({ }^{\Phi} N^{*} Y\right) \oplus H_{\mathrm{S}}^{n-i}\left({ }^{\Phi} N^{*} Y\right) \frac{d x}{x} & \text { if } j=n+1, \\ 0 & \text { otherwise. }\end{cases}
$$

Thus, ${ }^{\partial} E\left({ }^{\Phi} \mathcal{I}_{\partial}\right)$ degenerates at ${ }^{\partial} E^{2}$. The convergence of these spectral sequences follows from Corollary B.9. Now a map between filtered complexes that induces an isomorphism on $E^{\infty}$ is actually a quasi-isomorphism, provided the spectral sequences are convergent [24]. We summarize these remarks as follows.

Theorem 4.6. The map $\Theta$ induces an isomorphism

$$
\begin{aligned}
H H_{k}\left({ }^{\Phi} \mathcal{I}_{\partial}\right) \cong H H_{k}\left({ }^{s c} \mathcal{I}_{\partial}\right) & \cong H_{\mathrm{S}}^{2 n+2-k}\left({ }^{\Phi} N^{*} Y\right) \oplus H_{\mathrm{S}}^{2 n+1-k}\left({ }^{\Phi} N^{*} Y\right) \\
& \cong H^{n+1-k}(Y, \mathcal{O}(Y)) \oplus H^{n-k}(Y, \mathcal{O}(Y)),
\end{aligned}
$$

where $\mathcal{O}(Y) \rightarrow Y$ is the orientation bundle of $Y$. Moreover, $H_{k}\left({ }^{\Phi} \mathcal{I}_{\partial}\right)$ inherits the filtration by the total $x$-order, and

$$
H H_{k}\left({ }^{\Phi} \mathcal{I}_{\partial}\right)_{j}= \begin{cases}H H_{k}\left({ }^{s} \mathcal{I}_{\partial}\right), & \text { if } j \geq k-n-1, \\ 0 & \text { otherwise. }\end{cases}
$$

Proof. For the last isomorphism in (23) we have used the Thom isomorphism. 


\section{The homology of The Symbolic $\Phi$-Algebras}

As Benameur and Nistor noticed [2], the homology of the algebra of symbols of a large class of pseudodifferential algebras can be computed using the spectral sequence argument of Wodzicki [43] and Brylinski and Getzler [3], [4]. In fact, this spectral sequence degenerates at $E^{2}$. However, the convergence of this sequence is non-trivial because of the boundary filtration that plays a role in the definition of the Hochschild chain spaces.

For the algebra $\Psi^{\mathbb{Z}}(M)$, where $M$ is a closed manifold, the convergence is almost tautological (see, however, Appendix (B). It is also fairly obvious for the doubleedge algebra [14, because of the vanishing of the double-edge Poisson bracket at the boundary. In the fibered cusp case, however, convergence is subtle. We prove in Appendix B a general convergence result which applies to this case, formalizing the corresponding result from 35 .

The Hochschild chain spaces $C_{k}\left({ }^{\Phi} \mathcal{A}_{\sigma}\right)$ admit a filtration given by the total operator order of the factors in the tensor product. This filtration is compatible with the boundary map; hence it induces a spectral sequence ${ }^{\sigma} E$. The ${ }^{\sigma} E^{1}$ term is just the homology of the graded algebra, which in turn is isomorphic to a space of exterior forms, the isomorphism being given by the Hochschild-Kostant-Rosenberg map:

$$
{ }^{\sigma} E_{i, j}^{1}\left({ }^{\Phi} \mathcal{A}_{\sigma}\right)=H H_{i+j}\left(\Psi_{\Phi}^{[\mathbb{Z}], \mathbb{Z}}(X)\right)_{[i]} \simeq \Lambda_{[i]}^{i+j}\left({ }^{\Phi} T^{*} X \backslash\{0\}\right)\left[x^{-1}\right]
$$

where as usual the subscript $[i]$ in $\Lambda_{[i]}^{*}$ stands for the space of homogeneous forms of degree $i$ with respect to the natural $\mathbb{R}_{+}$-action along the fibers of ${ }^{\Phi} T^{*} X \backslash\{0\}$. We claim that up to sign the boundary map $d^{1}$ equals $*_{\Phi} d *_{\Phi}$, where $*_{\Phi}=\left(\iota_{\Phi}^{*}\right)^{-1} * \iota_{\Phi}^{*}$, and $*$ is the symplectic duality operator on $T^{*} X[3]$. Indeed, this can be proved as in [4], using the form of the Poisson bracket (5). In fact, this claim also follows from the corresponding result in 4 for the interior of $X$, and then by continuity at the boundary.

Notice now that $*_{\Phi}$ splits according to homogeneity:

$$
{ }^{*} \Phi: \Lambda_{[l]}^{k}\left({ }^{\Phi} T^{*} X \backslash\{0\}\right) \rightarrow \Lambda_{[N-k+l]}^{2 N-k}\left({ }^{\Phi} T^{*} X \backslash\{0\}\right)
$$

and that the de Rham cohomology of homogeneous forms is concentrated in homogeneity 0 . Indeed, on $\Lambda_{[p]}^{*}$, contraction with the radial vector field $\mathcal{R}$ in the fibers of ${ }^{\Phi} T^{*} X$ is a homotopy between $p I$ and 0 . It follows that

$$
{ }^{\sigma} E_{i, j}^{2}\left({ }^{\Phi} \mathcal{A}_{\sigma}\right)= \begin{cases}H^{N-i}\left({ }^{\Phi} S^{*} X \times S^{1}\right) \oplus H^{N-i-1}\left(\left.{ }^{\Phi} S^{*} X\right|_{\partial X} \times S^{1}\right) & \text { if } j=N \\ 0 & \text { otherwise }\end{cases}
$$

This proves in particular the degeneracy of the spectral sequence, because all the higher differentials $d^{k}$ for $k>1$ must vanish. We must prove convergence; that is, we must show that the ${ }^{\sigma} E^{\infty}$ terms are isomorphic to the graded groups associated to $H H\left({ }^{\Phi} \mathcal{A}_{\sigma}\right)$ with respect to the symbol filtration. This is unfortunately not obvious: the $E^{0}$ term of the spectral sequence has infinitely many nonzero components along the diagonals $\left\{(i, j) \in \mathbb{Z}^{2} ; i+j=\right.$ constant $\}$. So standard diagram chasing will produce infinite sums of Hochschild chains. Such sums make sense (i.e., are asymptotically summable) only if they are uniformly bounded in the three filtrations from Appendix B Moreover, as for any spectral sequence, we only get information about filtration quotients of the homology; we include in the definition 
of convergence the condition that the "residual" part of the homology (i.e., those classes that admit representatives of arbitrarily low filtration order) vanishes.

We are going to prove the convergence of ${ }^{\sigma} E\left({ }^{\Phi} \mathcal{A}_{\sigma}\right)$ using Theorem B.4 by showing that assumptions H1-H6 hold. First, the fibered cusp symbol algebra satisfies the hypothesis of Lemma B.6 so $\mathrm{H} 4$ and $\mathrm{H} 5$ do hold. H6 is obvious. Condition H2 follows immediately from (24), likewise the first part of condition $\mathrm{H} 1$ (the existence of $\beta \in C_{k}^{i+1, \mathbb{Z} ; \mathbb{Z}}$ such that $\left.b(\beta)+\alpha \in C_{k-1}^{i-1, \mathbb{Z} ; \mathbb{Z}}\right)$.

For H3, we study the spectral sequence ${ }^{\sigma} E$ (relative to the symbol filtration) of the graded algebra $\Phi_{\mathcal{A}}^{\mathbb{Z},[\mathbb{Z}]}$ of ${ }^{\Phi} \mathcal{A}_{\sigma}$ with respect to the boundary filtration. Recall from (92) that ${ }^{\Phi} \mathcal{A}_{\sigma}^{\mathbb{Z},[\mathbb{Z}]}$ is isomorphic to the algebra $\widetilde{\Psi}_{\operatorname{sus}\left({ }^{\Phi} N^{*} Y\right)-\varphi}^{\mathbb{Z}}(\partial X) \otimes \mathbb{C}\left[x, x^{-1}\right]$.

Proposition 5.1. The spectral sequence ${ }^{\sigma} E\left({ }^{\Phi} \mathcal{A}_{\sigma}^{\mathbb{Z},[\mathbb{Z}]}\right)$ of the Hochschild complex of ${ }^{\Phi} \mathcal{A}_{\sigma}^{\mathbb{Z},[\mathbb{Z}]}$ degenerates at ${ }^{\sigma} E^{2}$.

Proof. This result is essentially contained in [33]. Consider the short exact sequence of algebras

$$
0 \longrightarrow B_{\mathrm{sus}} \longrightarrow{ }^{\Phi} \mathcal{A}_{\sigma}^{\mathbb{Z},[\mathbb{Z}]} \longrightarrow F_{\text {sus }} \longrightarrow 0,
$$

where $B_{\text {sus }}$ is the ideal of those symbols in ${ }^{\Phi} \mathcal{A}_{\sigma}^{\mathbb{Z},[\mathbb{Z}]}$ that vanish rapidly at the vertical sub-bundle of ${ }^{\Phi} T^{*} X$, and (25) serves as the definition of the algebra $F_{\text {sus. }}$. This short exact sequence induces long exact sequences of ${ }^{\sigma} E^{1}$ terms, but these actually decompose as short exact sequences of homogeneous differential forms

$$
0 \longrightarrow{ }^{\sigma} E_{i j}^{1}\left(B_{\mathrm{sus}}\right) \longrightarrow{ }^{\sigma} E_{i j}^{1}\left({ }^{\Phi} \mathcal{A}_{\sigma}^{\mathbb{Z},[\mathbb{Z}]}\right) \longrightarrow{ }^{\sigma} E_{i j}^{1}\left(F_{\text {sus }}\right) \longrightarrow 0
$$

Thus we get long exact sequences both on the ${ }^{\sigma} E^{2}$ terms (from the short exact sequences of ${ }^{\sigma} E^{1}$ terms) and on Hochschild homology (induced from (25) by $\mathrm{H}$ unitality of $B_{\text {sus }}$ ).

There exist many exterior derivations on the algebra ${ }^{\Phi} \mathcal{A}_{\sigma}^{\mathbb{Z},[\mathbb{Z}]}$, namely the covariant derivatives $\nabla_{V}$ of Section 4 for $V$ either equal to $\partial_{x}$ or a vector field with polynomial coefficients on ${ }^{\Phi} N Y$. These derivations act on the Hochschild complex by contraction and by Lie derivative (see [20]). These actions descend to Hochschild homology and to the spectral sequences, and they commute with the differentials.

Since the polynomial functions $f$ on ${ }^{\Phi} N Y$ and in the variable $x$ are central in ${ }^{\Phi} \mathcal{A}_{\sigma}^{\mathbb{Z},[\mathbb{Z}]}$, we can define an "exterior product" action of such a function $f$ on the Hochschild complex by

$$
a_{0} \otimes \ldots \otimes a_{k} \stackrel{f \wedge}{\longmapsto} \sum_{i=0}^{k}(-1)^{i} a_{0} \otimes \cdots \otimes a_{i} \otimes f \otimes \cdots \otimes a_{k} .
$$

Again, this operation commutes with the Hochschild boundary map, and hence with all differentials in the spectral sequence. It is straightforward to check that, for a polynomial function $f$ as above,

$$
\left[e_{\nabla_{V}}, f \wedge\right]=V(f) I, \quad\left[L_{\nabla_{V}}, f \wedge\right]=V(f) \wedge, \quad f \wedge g \wedge=-g \wedge f \wedge
$$

where $V(f)$ is the $V$-derivative of the function $f$ in the direction of the vector $V$. Also, on Hochschild homology, $e_{\nabla_{U}} e_{\nabla_{V}}=-e_{\nabla_{V}} e_{\nabla_{U}}$. This was shown by hand in [35], but it follows directly from the product structure of Hochschild cohomology $H H^{*}(A, A)$ and its action on homology (we are grateful to Colin Ingalls for this argument). 
These algebraic properties of $\Phi_{\mathcal{A}_{\sigma}}^{\mathbb{Z},[\mathbb{Z}]}$ and the explicit computation of ${ }^{\sigma} E^{2}\left(F_{\text {sus }}\right)$ and ${ }^{\sigma} E^{2}\left(B_{\text {sus }}\right)$ similar to 33 are enough to show that (i) the spectral sequences ${ }^{\sigma} E\left(F_{\text {sus }}\right)$ and ${ }^{\sigma} E\left(B_{\text {sus }}\right)$ degenerate at ${ }^{\sigma} E^{2}$, and (ii) the boundary map in the sequence of ${ }^{\sigma} E^{2}$ terms vanishes. Inductively, we get a long exact sequence of ${ }^{\sigma} E^{p}$ terms that actually splits into short exact sequences, for $p \geq 1$. The Five Lemma shows that ${ }^{\sigma} E\left({ }^{\Phi} \mathcal{A}_{\sigma}^{\mathbb{Z},[\mathbb{Z}]}\right)$ also degenerates at ${ }^{\sigma} E^{2}$.

By Corollary B.8 we see that H3 is fulfilled. Now we use the notation of Appendix B to prove $\mathrm{H} 1$.

Proposition 5.2. Let $a \in C_{k}^{i, j ; l}\left({ }^{\Phi} \mathcal{A}_{\sigma}\right)$ be a chain surviving at ${ }^{\sigma} E_{i, k-i}^{2}$, i.e., such that $d^{0}[a]_{\sigma_{E^{0}}}=0$ and $d^{1}[a]_{\sigma_{E^{1}}}=0$. If $k-i \neq N$ and $l \geq 1+\max \left\{i+\delta_{i+1}^{0}, j+\delta_{j+1}^{0}\right\}$, then there exists $\beta \in C_{k}^{i+1, j+1 ; l}\left({ }^{\Phi} \mathcal{A}_{\sigma}\right)$ such that $b(\beta)+a \in C_{k}^{i, j+1 ; l}$ and $b(\beta)+a$ is exact as a chain in $C_{k}^{[i], j+1 ; l}$.

Proof. Let $[a]_{{ }_{\sigma} E^{1}}\left({ }^{\Phi} \mathcal{A}_{\sigma}\right)$ be the form represented by $a$ at ${ }^{\sigma} E_{i, k-i}^{1}\left({ }^{\Phi} \mathcal{A}_{\sigma}\right)$. Then $*_{\Phi}[a]_{\sigma E^{1}}$ is closed, since $a$ is a cycle, and it is exact because $k-i \neq N$. Moreover,

$$
(k-i-N) *_{\Phi}[a]_{\sigma_{E^{1}}}=d \imath_{\mathcal{R}} *_{\Phi}[a]_{\sigma_{E^{1}}}=d *_{\Phi} \iota_{\Phi}^{*}(\alpha) \wedge[a]_{\sigma_{E^{1}}},
$$

where $\alpha$ is the canonical 1 -form on $T^{*} X$. By Lemma B.7 the form $\iota_{\Phi}^{*}(\alpha) \wedge[a]_{\sigma_{E^{1}}}$ can be represented by a chain $\beta_{1} \in C_{k}^{[i+1], j+1 ; l}\left(\Psi_{\Phi}^{[\mathbb{Z}], \mathbb{Z}}\right)$.

Thus H1 also holds for ${ }^{\Phi} \mathcal{A}_{\sigma}$; so we can apply Theorem B.4. An entirely similar analysis is done for the algebras ${ }^{\Phi} \mathcal{A}_{\partial, \sigma}$ and ${ }^{\Phi} \mathcal{I}_{\sigma}$. We summarize the results in the next theorem. The subscripts rel (respectively, abs) denote de Rham cohomology of forms vanishing to the boundary (respectively, smooth up to the boundary).

Theorem 5.3.

$$
\begin{aligned}
H H_{k}\left({ }^{\Phi} \mathcal{I}_{\sigma}\right) & \cong H_{r e l}^{2 N-k}\left({ }^{\Phi} S^{*} X \times S^{1}\right) \\
H H_{k}\left({ }^{\Phi} \mathcal{A}_{\sigma}\right) & \cong H_{a b s}^{2 N-k}\left({ }^{\Phi} S^{*} X \times S^{1}\right) \oplus H^{2 N-1-k}\left(\left.{ }^{\Phi} S^{*} X\right|_{\partial X} \times S^{1}\right), \\
H H_{k}\left({ }^{\Phi} \mathcal{A}_{\partial, \sigma}\right) & \cong H^{2 N-k}\left(\left.{ }^{\Phi} S^{*} X\right|_{\partial X} \times S^{1} \times S^{1}\right) .
\end{aligned}
$$

Moreover, for $i \neq k-N, H H_{k}\left({ }^{\Phi} \mathcal{A}_{\sigma}\right)_{[i]}$ and $H H_{k}\left({ }^{\Phi} \mathcal{A}_{\partial, \sigma}\right)_{[i]}$ vanish, and $H H_{k}\left({ }^{\Phi} \mathcal{A}_{\sigma}\right)$ (respectively $\left.H_{k}\left({ }^{\Phi} \mathcal{A}_{\partial, \sigma}\right)\right)$ can be represented by chains in $C_{k}^{k-N, k-n-1}\left({ }^{\Phi} \mathcal{A}_{\sigma}\right)$ (resp. in $\left.C_{k}^{k-N, k-n-1}\left({ }^{\Phi} \mathcal{A}_{\partial, \sigma}\right)\right)$.

Proof. The isomorphisms follow from Theorem B.4 By analyzing the $\Phi$-symplectic duality operator $*_{\Phi}$ (the homogeneities given in (22) are valid for absolute forms with symbol coefficients as well) and by Lemma B.7, we get the indices with respect to the boundary filtration as claimed.

We remark that the long exact sequence in Hochschild homology (which exists since ${ }^{\Phi} \mathcal{I}_{\sigma}$ is $H$-unital) coincides with the long exact sequence of ${ }^{\sigma} E^{2}$ terms induced from the short exact sequence of ${ }^{\sigma} E^{1}$ terms, which is just the de Rham absoluterelative cohomology long exact sequence.

\section{The Boundary SEquence}

In order to compute $H H\left({ }^{\Phi} \mathcal{A}_{\partial}\right)$, consider the short exact sequence of algebras

$$
0 \rightarrow{ }^{\Phi} \mathcal{I}_{\partial} \rightarrow{ }^{\Phi} \mathcal{A}_{\partial} \rightarrow{ }^{\Phi} \mathcal{A}_{\partial, \sigma} \rightarrow 0
$$


We have already computed the homologies of the two extremal algebras. By $H$ unitality of ${ }^{\Phi} \mathcal{I}_{\partial}$ (an issue that we are not going to stress here), there exists a long exact sequence in Hochschild homology induced from (26). This long exact sequence is completely determined by the boundary maps

$$
\delta_{k}: H H_{k}\left({ }^{\Phi} \mathcal{A}_{\partial, \sigma}\right) \rightarrow H H_{k-1}\left({ }^{\Phi} \mathcal{I}_{\partial}\right) .
$$

When looking at the associated graded algebras with respect to the boundary filtration, the result is quite simple, in the sense that all boundary maps vanish. We have already mentioned this fact in Proposition 5.1. This says basically (as shown in [33]) that there is no analog of the index map for suspended operators. It follows that the boundary maps $\delta$ decrease the boundary filtration order by at least 1 . The only relevant dimensions are for $k \leq n+2$; otherwise $H H_{k-1}\left({ }^{\Phi} \mathcal{I}_{\partial}\right)=0$. Now note that $H H_{k+1}\left({ }^{\Phi} \mathcal{A}_{\partial, \sigma}\right)$ has representatives in boundary filtration $k-n$ (Theorem 5.3).

Let $\alpha$ be a homology class in $H H_{k+1}\left({ }^{\top} \mathcal{A}_{\partial, \sigma}\right)$. By $H$-unitality, there exists $A \in$ $C_{k+1}\left({ }^{\Phi} \mathcal{A}_{\partial, \sigma}\right)$ representing the class $\alpha$ such that $b(A) \in C_{k}\left({ }^{\Phi} \mathcal{I}_{\partial}\right)$. Moreover, we can assume that $A \in C_{k+1}^{N-k-1, k-n}\left({ }^{\Phi} \mathcal{A}_{\partial, \sigma}\right)$. From the fact that the boundary map in the associated graded algebras vanishes, we can also assume that $b(A) \in C_{k}^{k-n-1}\left({ }^{\Phi} \mathcal{I}_{\partial}\right)$.

The boundary map $\delta$ is defined simply by $\delta(\alpha):=[b(A)]$. We do not know in general how the isomorphism from Theorem 4.6 associates a cohomology class to a cycle; however, we have been able to show that the boundary filtration order of the cycle $b(A)$ is $k-n-1$, which is exactly the filtration order where $H H_{k}\left({ }^{\Phi} \mathcal{I}_{\partial}\right)$ is concentrated (Theorem 4.6). This means that $[b(A)]$ can be explicitly computed from its top component:

$$
[b(A)]=*_{s c} K\left(b(A)_{[k-n-1]}\right) \in H_{\mathrm{S}}^{2 n+2-k}\left({ }^{\Phi} N Y \otimes \mathcal{L}\right) .
$$

Now use the Thom isomorphism between Schwartz-coefficient de Rham cohomology and cohomology of the base with coefficients in the orientation bundle:

$$
[b(A)]=\int_{\Phi N Y / Y} *_{s c} K\left(b(A)_{[k-n-1]}\right) \in H^{n+1-k}(Y \otimes \mathcal{L}, \mathcal{O}(Y)) .
$$

Now let $Q$ be a positive $\Phi$-operator of order $(1,0)$ and let $Q^{z}$ be its complex powers. The construction of complex powers in the $\Phi$-setting is briefly discussed in Theorem 7.1. Notice the identity

$$
Q^{z} b(A)=b\left(Q^{z} A\right)+z Q^{z} e_{\log Q}(A)+O\left(z^{2}\right),
$$

where $e_{\log Q}(A)$ is the action of the exterior derivation $A_{k} \mapsto\left[\log Q, A_{k}\right]$ on the Hochschild complex given explicitly by (see also [20])

$$
A_{0} \otimes \ldots \otimes A_{k} \mapsto\left[\log Q, A_{k}\right] A_{0} \otimes \ldots \otimes A_{k-1} .
$$

We can then write

$$
\begin{aligned}
{[b(A)]=} & \int_{\Phi N Y / Y} *_{s c} K\left(Q^{z} b(A)_{[k-n-1]}\right)_{\mid z=0} \\
= & {\left[\int_{\Phi N Y / Y} *_{s c} K\left(b\left(Q^{z} A\right)_{[k-n-1]}\right)_{\mid z=0}\right]_{[0]} } \\
& +\operatorname{Res}_{z=0}\left[\int_{\Phi N Y / Y} *_{s c} K\left(Q^{z} e_{\log Q}(A)\right)\right]_{[0]},
\end{aligned}
$$


where the subscript [0] denotes the part of homogeneity 0 in $x$. We first examine the second term in (28). By Fubini's formula, it equals

$$
-\frac{1}{(2 \pi)^{m}}\left[\operatorname{Res}_{z=0} \int_{\Phi T^{*} X / Y} *_{\phi} H K R\left(Q^{z} e_{\log Q}(A)\right)\right]_{[0]} .
$$

We claim that this is just

$$
\frac{1}{(2 \pi)^{m}} \int_{\Phi^{*} X / Y} \alpha
$$

Indeed, $*_{\phi} H K R\left(Q^{z} e_{\log Q}(A)\right)$ is a form with symbol coefficients on ${ }^{\Phi} T^{*} X$ of homogeneity $z$. So the residue only depends on the principal symbol and is given by the above formula by a standard computation in Wodzicki-type formulas.

We claim now that the first term in (28) vanishes in cohomology, i.e., it is exact. This would be clear if $b\left(Q^{z} A\right)_{[k-n]}$ were equal to 0 , since in that case the same computation that gives $d^{1}$ in ${ }^{\partial} E\left({ }^{\Phi} \mathcal{I}_{\partial}\right)$ would give

$$
*_{s c} K\left(b\left(Q^{z} A\right)_{[k-n-1]}\right)=d *_{s c} K\left(Q^{z} A\right)_{[k-n]} .
$$

This holds for values of $z$ of small enough real part. By the Hodge theorem, the space of exact forms is closed in the $\mathcal{C}^{\infty}$ topology; hence the regularized value at $z=0$ is also exact. In general, $b\left(Q^{z} A\right)_{[k-n]} \equiv 0$ modulo an entire family of chains of order $(N-k-2+z,[k-n])$ that vanishes at $z=0$; hence the identity (29) is shown to be valid at $z=0$ as in [35. We summarize what we have obtained so far.

Proposition 6.1. In terms of the identifications of Theorems 5.3 and 4.6, the boundary map (27) is given by

$$
\delta_{k}=\frac{1}{(2 \pi)^{m}} \int_{\Phi S^{*} X / Y} .
$$

The two copies of $S^{1}$ in Theorem 5.3 correspond to a concentrated way of writing the de Rham cohomology of homogeneous forms in the variables $x$ and $r$. By Proposition 6.1 the boundary map sends $H^{2 N-k-1}\left(\left.{ }^{\Phi} S^{*} X\right|_{\partial X} \times S^{1}\right) \otimes \frac{d r}{r}$ to 0 ; hence (from the long exact sequence induced by (26)) this space lies in the image of the canonical map $H H_{k}\left({ }^{\Phi} \mathcal{A}_{\partial}\right) \rightarrow H H_{k}\left({ }^{\Phi} \mathcal{A}_{\partial, \sigma}\right)$. The dimension of the sphere fibers of the fibration $\left.{ }^{\Phi} S^{*} X\right|_{\partial X} \rightarrow \partial X$ equals the dimension of the base, and so from the Leray spectral sequence it follows that $\left.{ }^{\Phi} S^{*} X\right|_{\partial X}$ is cohomologically a product:

$$
H^{*}\left(\left.{ }^{\Phi} S^{*} X\right|_{\partial X}\right) \simeq H^{*}(\partial X, \mathcal{O}(X)) \otimes H^{*}\left(S^{N-1}\right) .
$$

For $k \leq n+2 \leq N$ we deduce that

$$
H^{2 N-k}\left(\left.{ }^{\Phi} S^{*} X\right|_{\partial X} \times S^{1}\right) \simeq H^{N-k+1}\left(\partial X \times S^{1}, \mathcal{O}(X)\right) .
$$

We get from here a characterization of $H H\left({ }^{\Phi} \mathcal{A}_{\partial}\right)$. Let $I_{N-k+1}$ be the push-forward map (integral along the fiber)

$$
I_{N-k+1}: H^{N-k+1}\left(\partial X \times S^{1}, \mathcal{O}(X)\right) \rightarrow H^{n-k+2}\left(Y \times S^{1}, \mathcal{O}(Y)\right) .
$$

\section{Theorem 6.2.}

$$
H H_{k}\left({ }^{\Phi} \mathcal{A}_{\partial}\right) \cong H^{2 N-k-1}\left(\left.{ }^{\Phi} S^{*} X\right|_{\partial X} \times S^{1}\right) \oplus \operatorname{ker}\left(I_{N-k+1}\right) \oplus \operatorname{coker}\left(I_{N-k}\right) .
$$

Proof. In the long exact sequence induced by (26) we write $H H_{k}\left({ }^{\Phi} \mathcal{A}_{\partial}\right)$ as $\operatorname{ker}\left(\delta_{k}\right) \oplus$ $\operatorname{coker}\left(\delta_{k+1}\right)$. These spaces were identified above. 
Corollary 6.3. The dimension of $H H_{0}\left({ }^{\Phi} \mathcal{A}_{\partial}\right)$ is 1 , and so there exists a unique (up to a constant) continuous trace on ${ }^{\Phi} \mathcal{A}_{\partial}$.

Proof. From Theorem 6.2 for $k=0$ we get

$$
\begin{aligned}
H^{2 N-k-1}\left(\left.{ }^{\Phi} S^{*} X\right|_{\partial X} \times S^{1}\right) & =\mathbb{C}, \\
I_{N+1} & =0, \\
\operatorname{coker}\left(I_{N}\right) & =0 .
\end{aligned}
$$

Only the last equality needs some explanation. The integral map

$$
I_{\partial X}: H^{N}\left(\partial X \times S^{1}, \mathcal{O}(X)\right) \rightarrow \mathbb{C}
$$

is an isomorphism. The same is true for $I_{Y}: H^{n+1}\left(Y \times S^{1}, \mathcal{O}(Y)\right) \rightarrow \mathbb{C}$. But $I_{\partial X}=I_{Y} \circ I_{N} ;$ so $I_{N}$ is also an isomorphism.

We remark that the boundary map and the homology of $\Phi^{\Phi} \mathcal{A}_{\partial}$ depend strongly on the geometry of the boundary fibration.

\section{Traces And generalized Residues of THE Fibered Cusp CAlCulus}

The computations of the previous sections show that $\operatorname{dim}_{\mathbb{C}} H H_{0}(A)=1$ for $A={ }^{\Phi} \mathcal{I}_{\sigma},{ }^{\Phi} \mathcal{I}_{\partial},{ }^{\Phi} \mathcal{A}_{\sigma},{ }^{\Phi} \mathcal{A}_{\partial},{ }^{\Phi} \mathcal{A}_{\partial, \sigma}$; thus, there exists, up to a multiplicative constant, a unique trace on these algebras. We are going to identify these traces as residues of the analytic continuation of a "double-zeta" function, and we are going to give, additionally, explicit formulas. The corresponding results about traces for the cusp calculus were obtained in [34. Section 5]. Some of the proofs depend on formal manipulations with zeta functions that can be found in [34]; so we will only state those results and leave the necessary changes of the proofs to the reader.

Theorem 7.1. Let $Q \in \Psi_{\Phi}^{1,0}(X)$ be a positive $\Phi$-operator, possibly with bundle coefficients. Then the family of complex powers $\mathbb{C} \ni z \mapsto Q^{z}$ is a holomorphic family of $\Phi$-operators, $Q^{z} \in \Psi_{\Phi}^{z, 0}(X)$.

Proof. The complex powers of $Q$ are constructed using the method of Bucicovschi [5], who extended the proof of Guillemin [12] Theorem 5.5] to the case of noncommutative symbols. We assume the reader to be familiar with these two papers for the purpose of this proof.

The algebra $\Psi_{\Phi}^{m}(X)$ has two symbol maps, which must be treated simultaneously. We first note that the complex powers of $\mathcal{N}_{\Phi}(Q)$ form a holomorphic family of suspended operators, $\mathcal{N}_{\Phi}(Q)^{z} \in \Psi_{\operatorname{sus}\left({ }^{\Phi} N Y\right)-\varphi}^{z}(\partial X)$. This follows modulo $\Psi_{\left.\operatorname{sus}^{(}{ }^{(} N Y\right)-\varphi}^{-\infty}(\partial X)$ from [5, Proposition 1.4], and is corrected to a true family of complex powers as in [12, Section 5]. It is clear that the principal (conormal) symbol of $Q$ admits complex powers, and that

$$
\sigma_{z}\left(\mathcal{N}_{\Phi}(Q)^{z}\right)=\left.\sigma_{1}(Q)^{z}\right|_{\partial X}
$$

This allows us to start Bucicovschi's induction argument: there exists a holomorphic family $Q_{0}(z)$ with $Q_{0}(0)=I, Q_{0}(1)=Q, \sigma_{z}\left(Q_{0}(z)\right)=\sigma_{1}(Q)^{z}$ and $\mathcal{N}_{\Phi}\left(Q_{0}(z)\right)=$ $\mathcal{N}_{\Phi}(Q)^{z}$. This implies that

$$
Q_{0}(z) Q_{0}(\tau) Q_{0}(z+\tau)^{-1}=1+R_{1}(z, \tau),
$$

where $R_{1}(z, \tau) \in \Psi_{\Phi}^{-1,-1}(X)$ is holomorphic in the two variables. Notice that in [5] the error symbols $R_{j}$ are sections in a von Neumann algebra bundle, whereas 
here they have a component in the Fréchet algebra $\Psi_{\operatorname{sus}\left({ }^{\Phi} N Y\right)-\varphi}^{0}(\partial X)$ (the boundary symbol). Nevertheless, there is basically no modification needed to prove 5 . Propositions $1.3,1.4$ ] in our case; a new argument is only necessary to show that the map

$$
\Psi_{\operatorname{sus}\left({ }^{\Phi} N Y\right)-\varphi}^{k}(\partial X) \ni A \mapsto \int_{0}^{1} Q^{-t} A Q^{t} d t \in \Psi_{\operatorname{sus}\left({ }^{\Phi} N Y\right)-\varphi}^{k}(\partial X)
$$

is surjective. This surjectivity is true modulo $\Psi_{\operatorname{sus}\left({ }^{\Phi} N Y\right)-\varphi}^{k-1}(\partial X)$ (it reduces then to Bucicovschi's case of matrix-valued symbols), and also on $\Psi_{\operatorname{sus}\left(\Phi_{N Y}{ }^{-\infty}-\varphi\right.}(\partial X)$. Asymptotic completeness of $\Psi_{\operatorname{sus}\left({ }^{\Phi} N Y\right)-\varphi}^{0}(\partial X)$ modulo the ideal $\Psi_{\operatorname{sus}\left({ }^{\Phi} N Y\right)-\varphi}^{-\infty}(\partial X)$ ends the proof of the induction step.

By induction and asymptotic completeness of $\Psi_{\Phi}(X)$ modulo $\Psi_{\Phi}^{-\infty,-\infty}(X)$, we get an approximate complex powers family $Q(z)$ modulo $\Psi_{\Phi}^{-\infty,-\infty}(X)$, i.e.,

$$
Q(z) Q(\tau) Q(z+\tau)^{-1}=1+R(z, \tau) \in 1+\Psi_{\Phi}^{-\infty,-\infty}(X) .
$$

Again as in [12 Section 5] we show that $Q(z)$ differs from the true complex powers family $Q^{z}$ by a holomorphic family in $\Psi_{\Phi}^{-\infty,-\infty}(X)$; so it follows that $Q^{z} \in \Psi_{\Phi}^{z, 0}(X)$ is a holomorphic family.

In particular, we see that the complex power $Q^{z}$ is again a $\Phi$-operator of complex order $z \in \mathbb{C}$. Extending the approach of Guillemin [12] and Bucicovschi [5], complex powers could recently be constructed simultaneously for a large class of boundary fibration structures and non-compact manifolds 11; however, it is necessary to stress that in this generality complex powers are realized in the so-called Guillemin completion of the calculus, which contains slightly more smoothing operators than the original (small) calculus.

Proposition 7.2. Let $\Omega \subseteq \mathbb{C}^{2}$ be open and connected with $[R, \infty) \times[R, \infty) \subseteq \Omega$ for some $R \in \mathbb{R}$, and let $A: \Omega \longrightarrow \Psi_{\Phi}^{m_{0}, k_{0}}(X)$ be holomorphic. Then the functions

$$
T_{k}: \Omega \cap\left\{\operatorname{Re} z>k_{0}+n+1\right\} \cap\left\{\operatorname{Re} \lambda>m_{0}+N\right\} \longrightarrow \mathbb{C},
$$

with $T_{1}:(\lambda, z) \mapsto \operatorname{Tr}\left(A(\lambda, z) \varrho_{N}^{z} Q^{-\lambda}\right)$ and $T_{2}:(\lambda, z) \mapsto \operatorname{Tr}\left(A(\lambda, z) Q^{-\lambda} \varrho_{N}^{z}\right)$, are holomorphic. Moreover, there exist meromorphic functions $\widetilde{T}_{k}: \Omega \longrightarrow \mathbb{C}$ with at most simple poles at $\lambda=m_{0}+N-\ell, \ell \in \mathbb{N}_{0}$, and $z=k_{0}+n+1-j, j \in \mathbb{N}_{0}$, which coincide with $T_{k}$ on the component of $\Omega$ containing $\left[R^{\prime}, \infty\right) \times\left[R^{\prime}, \infty\right)$ for some $R^{\prime} \in \mathbb{R}$.

For $A \in \Psi_{\Phi}^{m_{0}, k_{0}}(X)$, we let $Z_{\varrho_{N}, Q}(A)$ be the meromorphic extension of the holomorphic function

$$
\left\{\operatorname{Re} z>k_{0}+n+1\right\} \cap\left\{\operatorname{Re} \lambda>m_{0}+N\right\} \ni(\lambda, z) \longmapsto \operatorname{Tr}\left(A \varrho_{N}^{z} Q^{-\lambda}\right)
$$

which exists by Proposition 7.2 ; then $(\lambda, z) \mapsto z \lambda Z_{\varrho_{N}, Q}(A)(\lambda, z)$ is regular in a neighborhood of $0 \in \mathbb{C}^{2}$, and we can define $\operatorname{Tr}_{\partial, \sigma}(A), \widehat{\operatorname{Tr}}_{\partial}(A)$ and $\widehat{\operatorname{Tr}}_{\sigma}(A)$ by

$$
\begin{aligned}
z \lambda Z_{\varrho_{N}, Q}(A)(\lambda, z)=: & \operatorname{Tr}_{\partial, \sigma}(A)+\lambda \widehat{\operatorname{Tr}}_{\partial}(A)+z \widehat{\operatorname{Tr}}_{\sigma}(A) \\
& +\lambda^{2} W(\lambda, z)+\lambda z W^{\prime}(\lambda, z)+z^{2} W^{\prime \prime}(\lambda, z),
\end{aligned}
$$

where $W, W^{\prime}, W^{\prime \prime}$ are holomorphic near $0 \in \mathbb{C}^{2}$ (and not unique!). Because of

$$
\operatorname{Tr}\left(A \varrho_{N}^{z} Q^{-\lambda}-A Q^{-\lambda} \varrho_{N}^{z}\right)=\operatorname{Tr}\left(A\left(\mathrm{id}-Q^{-\lambda} \varrho_{N}^{z} Q^{\lambda} \varrho_{N}^{-z}\right) \varrho_{N}^{z} Q^{-\lambda}\right),
$$

we could have used in (30) also the zeta function $\widetilde{Z}_{\varrho_{N}, Q}(A):(\lambda, z) \mapsto \operatorname{Tr}\left(A Q^{-\lambda} \varrho_{N}^{z}\right)$. 
Furthermore, let $\omega_{\Phi}$ be the canonical singular symplectic form on ${ }^{\Phi} T^{*} X$, and let us denote by $i_{\mathcal{R}}$ (resp. $i_{x^{2} \partial_{x}}$ ) the contraction with the radial vector field $\mathcal{R}$ in the fibers of ${ }^{\Phi} T^{*} X$ (resp. with the canonical vector field $x^{2} \partial_{x}$, normal to the boundary). Recall that $\varrho_{\sigma}:{ }^{\Phi} \bar{T}^{*} X \rightarrow \overline{\mathbb{R}}_{+}$denotes a defining function for the $\Phi$-cosphere bundle ${ }^{\Phi} S^{*} X$. Moreover, the pull-back of the boundary defining function $\varrho_{N}: X \rightarrow \overline{\mathbb{R}}_{+}$ under the projection $\Phi \bar{T}^{*} X \rightarrow \overline{\mathbb{R}}_{+}$yields a defining function for the face $\left.{ }^{\Phi} \bar{T}^{*} X\right|_{\partial X}$, again denoted by $\varrho_{N}$ for simplicity.

In the rest of this section, we summarize the properties of the functionals $\operatorname{Tr}_{\partial, \sigma}$, $\widehat{\operatorname{Tr}}_{\partial}$, and $\widehat{\operatorname{Tr}}_{\sigma}$.

Proposition 7.3. The double-residue $\operatorname{Tr}_{\partial, \sigma}: \Psi_{\Phi}^{\mathbb{Z}, \mathbb{Z}}(X) \rightarrow \mathbb{C}$ is a trace functional that is independent of the choice of $Q$. Moreover,

$$
\operatorname{Tr}_{\partial, \sigma}\left(\Psi_{\Phi}^{-\infty, \mathbb{Z}}(X)+\Psi_{\Phi}^{\mathbb{Z},-\infty}(X)\right)=0
$$

Thus, $\operatorname{Tr}_{\partial, \sigma}$ defines a trace functional on ${ }^{\Phi} \mathcal{A}_{\partial, \sigma}$. If $A \in{ }^{\Phi} \mathcal{A}_{\partial, \sigma}$ is represented by $\sum_{k \leq k_{0}, m \leq m_{0}} A_{m, k}$ with $A_{m, k} \in \varrho_{N}^{-k} \varrho_{\sigma}^{-m} \mathcal{C}^{\infty}\left(\left.{ }^{\Phi} S^{*} X\right|_{\partial X}\right)$, then

$$
\operatorname{Tr}_{\partial, \sigma}(A)=(2 \pi)^{-N} \int_{\left.\Phi S^{*} X\right|_{\partial X}} A_{-N,-(1+n)} i_{x^{2} \partial_{x}} i_{\mathcal{R}} \omega_{\Phi}^{N}
$$

The "unique" traces on ${ }^{\Phi} \mathcal{A}_{\sigma}$ (resp. ${ }^{\Phi} \mathcal{A}_{\partial}$ ) are then given by the composition of $\operatorname{Tr}_{\partial, \sigma}$ with the natural projections ${ }^{\Phi} \mathcal{A}_{\sigma} \rightarrow{ }^{\Phi} \mathcal{A}_{\partial, \sigma}\left(\right.$ resp. $\left.{ }^{\Phi} \mathcal{A}_{\partial} \rightarrow{ }^{\Phi} \mathcal{A}_{\partial, \sigma}\right)$.

Since $Z_{Q, x}(A)$ is entire for $A \in \Psi_{\Phi}^{-\infty,-\infty}(X)$, the linear functionals $\widehat{\operatorname{Tr}}_{\sigma}$ and $\widehat{\operatorname{Tr}}_{\partial}$ descend to linear functionals on $\Psi_{\Phi}^{\mathbb{Z}, \mathbb{Z}} / \Psi_{\Phi}^{-\infty,-\infty}$.

Proposition 7.4. The restriction $\operatorname{Tr}_{\sigma}:{ }^{\Phi} \mathcal{I}_{\sigma} \rightarrow \mathbb{C}$ of $\widehat{\operatorname{Tr}}_{\sigma}$ to ${ }^{\Phi} \mathcal{I}_{\sigma}$ is a trace functional; it is independent of the choice of $Q$, and extends the Wodzicki residue for the double $2 X$ of $X$ by continuity from operators whose kernels are supported in the interior. If $A \in{ }^{\Phi} \mathcal{I}_{\sigma}$ has the asymptotic expansion $\sum_{m \leq m_{0}} A_{m}$ with $A_{m} \in$ $\varrho_{N}^{\infty} S^{[m]}\left({ }^{\Phi} T^{*} X\right)$, i.e., $a_{m}:=\left.\left(\varrho_{\sigma}^{m} A_{m}\right)\right|_{\Phi} S^{*} X \in \varrho_{N}^{\infty} \mathcal{C}^{\infty}\left({ }^{\Phi} S^{*} X\right)$, then

$$
\operatorname{Tr}_{\sigma}(A)=(2 \pi)^{-N} \int_{\Phi S^{*} X} a_{-N^{-}} i_{\mathcal{R}} \omega_{\Phi}^{N}
$$

To understand the functional $\widehat{\operatorname{Tr}}_{\sigma}$, we need to extend the integral

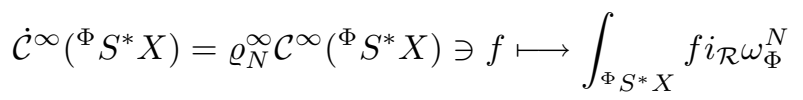

to functions $f \in \varrho_{N}^{k} \mathcal{C}^{\infty}\left({ }^{\Phi} S^{*} X\right)$ with finite $k \in \mathbb{Z}$. For such an $f \in \varrho_{N}^{k} \mathcal{C}^{\infty}\left({ }^{\Phi} S^{*} X\right)$, let

$$
H_{\varrho_{N}}(f):\{z \in \mathbb{C}: \operatorname{Re} z>n+1-k\} \longrightarrow \mathbb{C}: z \longmapsto \int_{\Phi^{*} X} \varrho_{N}^{z} f i_{\mathcal{R}} \omega_{\Phi}^{N} .
$$

Then $H_{\varrho_{N}}(f)$ is holomorphic and admits a meromorphic extension $H_{\varrho_{N}}(f)$ to the complex plane with at most simple poles at $z=n+1-k-j, j \in \mathbb{N}_{0}$. Let $\Phi-\int_{\Phi S^{*} X}^{\varrho_{N}} f i_{\mathcal{R}} \omega_{\Phi}^{N}$ be the regularized value of $H_{\varrho_{N}}(f)$ at $z=0$. Note that ${ }^{\Phi-} \int_{\Phi}^{\varrho_{N}} S^{*}$ extends (31) as desired to $\varrho_{N}^{k} \mathcal{C}^{\infty}\left({ }^{\Phi} S^{*} X\right)$, but depends mildly on the choice of the defining function $\varrho_{N}$.

Proposition 7.5. Let $A \in \Psi_{\Phi}^{m_{0}, k_{0}}(X)$ be arbitrary, and suppose that the residue class $A+\Psi_{\Phi}^{-\infty, \mathbb{Z}}(X)$ corresponds to $\sum_{m \leq m_{0}} A_{m}$ with $A_{m} \in \varrho_{N}^{-k_{0}} S^{[m]}\left({ }^{\Phi} T^{*} X\right)$, i.e., 


$$
\begin{aligned}
a_{m}:=\left.\left(\varrho_{\sigma}^{m} A_{m}\right)\right|_{\Phi_{S^{*} X}} \in & \varrho_{N}^{-k_{0}} \mathcal{C}^{\infty}\left({ }^{\Phi} S^{*} X\right) . \text { Then } \\
& \widehat{\operatorname{Tr}}_{\sigma}(A)=\frac{1}{(2 \pi)^{N}}{ }^{\Phi-} \int_{\Phi S^{*} X}^{\varrho_{N}} a_{-N} i_{\mathcal{R}} \omega_{\Phi}^{N} .
\end{aligned}
$$

Proof. Consider $A \varrho_{N}^{z}$ for $\operatorname{Re} z>k_{0}+n+1$. Then the function

$$
G_{\varrho_{N}}:\left\{z \in \mathbb{C}: \operatorname{Re} z>k_{0}+n+1\right\} \longrightarrow \mathbb{C}: z \mapsto \widehat{\operatorname{Tr}}_{\sigma}\left(A \varrho_{N}^{z}\right)
$$

is holomorphic, admits a meromorphic extension $G_{\varrho_{N}}$ to the complex plane with at most simple poles at $z=k_{0}+n+1-j, j \in \mathbb{N}_{0}$, and from (30) we see that $\widehat{\operatorname{Tr}}_{\sigma}(A)$ coincides with the regularized value of $G_{\varrho_{N}}$ at $z=0$. Exactly as in Proposition [7.4 we obtain $G_{\varrho_{N}}(z)=(2 \pi)^{-N} \int_{\Phi^{*} X} b(z)_{-N} i_{\mathcal{R}} \omega_{\Phi}^{N}$, where the residue class of $A \varrho_{N}^{z} \in$ $\Psi_{\Phi}^{m_{0}, k_{0}-\operatorname{Re} z}(X)$ modulo $\Psi_{\Phi}^{-\infty, \mathbb{Z}}(X)$ is represented by the sum $\sum_{m \leq m_{0}} B(z)_{m}$ with $B(z)_{m} \in \varrho_{N}^{\operatorname{Re} z-k_{0}} S^{[m]}\left({ }^{\Phi} T^{*} X\right)$ and $b(z)_{m}=\left.\left(\varrho_{\sigma}^{m} B(z)_{m}\right)\right|_{\Phi^{*}} S^{*}$. Since on the other hand we have $b(z)_{m}=\varrho_{N}^{z} a_{m}$, a further look at the definition of the $\Phi$-integral $\Phi-\int^{\varrho_{N}}$ completes the proof.

Finally, for the functional $\widehat{\operatorname{Tr}}_{\partial}$ we need to consider an extension of the canonical trace $\overline{\operatorname{Tr}}$ on the space $\widetilde{\Psi}_{\operatorname{sus}\left({ }^{\Phi} N^{*} Y\right)-\varphi}^{-\infty}(\partial X)$ to $\widetilde{\Psi}_{\operatorname{sus}\left({ }^{\Phi} N^{*} Y\right)-\varphi}^{M}(\partial X)$ for arbitrary finite $M \in \mathbb{Z}$ (recall the canonical trace Tr that was introduced in Section 2). To this end, fix a positive operator $Q_{\partial X} \in \widetilde{\Psi}_{\operatorname{sus}\left(\Phi^{*} Y\right)-\varphi}^{1}(\partial X)$. and construct the holomorphic family $\mathbb{C} \ni \lambda \mapsto Q_{\partial X}^{\lambda} \in \widetilde{\Psi}_{\operatorname{sus}\left({ }^{\Phi} N^{*} Y\right)-\varphi}(\partial X)$ of complex powers. Note that we could take $Q_{\partial X}=\mathcal{N}_{\Phi}(Q)$ with $Q_{\partial X}^{\lambda}=\mathcal{N}_{\Phi}(Q)^{\lambda}=\mathcal{N}_{\Phi}\left(Q^{\lambda}\right)$ for $Q \in \Psi_{\Phi}^{1,0}(X)$, as above. Then, for $\widetilde{h} \in \widetilde{\Psi}_{\operatorname{sus}\left({ }^{\Phi} N^{*} Y\right)-\varphi}^{M}(\partial X)$, the function $F_{Q_{\partial X}}(\widetilde{h}): \lambda \mapsto \overline{\operatorname{Tr}}\left(\widetilde{h} Q_{\partial X}^{-\lambda}\right)$ is holomorphic in the domain $\{\lambda \in \mathbb{C}: \operatorname{Re} \lambda>M+N\}$, and admits a meromorphic extension to the whole plane with at most simple poles at $\lambda=M+N-j, j \in \mathbb{N}_{0}$. Let us denote by $\overline{\operatorname{Tr}}_{Q_{\partial X}}(\widetilde{h})$ the regularized value of $F_{Q_{\partial X}}(\widetilde{H})$ at $\lambda=0$. By the very definition, $\overline{\operatorname{Tr}}_{Q_{\partial X}}$ is an extension of $\overline{\operatorname{Tr}}$ that, however, depends on the choice of the family $Q_{\partial X}$.

Proposition 7.6. The restriction $\operatorname{Tr}_{\partial}:{ }^{\Phi} \mathcal{I}_{\partial} \rightarrow \mathbb{C}$ of $\widehat{\operatorname{Tr}}_{\partial}$ to ${ }^{\Phi} \mathcal{I}_{\partial}$ is a trace functional; it does not depend on the choice of $Q$. If $A \in{ }^{\Phi} \mathcal{I}_{\partial}$ corresponds to $\sum_{k \leq k_{0}} \varrho_{\mathrm{ff}_{\Phi}}^{-k} \widetilde{A}_{k}$ with $\widetilde{A}_{k} \in \widetilde{\Psi}_{\operatorname{sus}\left({ }^{\Phi} N^{*} Y\right)-\varphi}^{-\infty}(\partial X)$, then

$$
\operatorname{Tr}_{\partial}(A)=\overline{\operatorname{Tr}}\left(\widetilde{A}_{-(n+1)}\right) .
$$

For fibered cusp operators $A$ of arbitrary order that vanish sufficiently fast at the front face, we also have a nice formula for $\widehat{\operatorname{Tr}}_{\partial}(A)$.

Proposition 7.7. Let $A \in \Psi_{\Phi}^{m_{0},-(n+1)}(X)$ be arbitrary, and suppose that the class $A+\Psi_{\Phi}^{\mathbb{Z},-\infty}(X)$ is represented by $\sum_{k \leq-(n+1)} \varrho_{\mathrm{ff}_{\Phi}}^{-k} \widetilde{A}_{k}$ with $\widetilde{A}_{k} \in \widetilde{\Psi}_{\operatorname{sus}\left({ }^{\Phi} N^{*} Y\right)-\varphi}^{m_{0}}(\partial X)$. Then

$$
\widehat{\operatorname{Tr}}_{\partial}(A)=\overline{\operatorname{Tr}}_{Q_{\partial X}}\left(\widetilde{A}_{-(n+1)}\right) \text {, }
$$

where $Q_{\partial X}=\mathcal{N}_{\Phi}(Q)$.

Proof. Consider $A Q^{-\lambda}$ for $\lambda \in \mathbb{C}$ with $\operatorname{Re} \lambda>m_{0}+N$. Then the function

$$
H_{Q}:\left\{\lambda \in \mathbb{C}: \operatorname{Re} \lambda>m_{0}+N\right\} \longrightarrow \mathbb{C}: \lambda \longmapsto \widehat{\operatorname{Tr}}_{\partial}\left(A Q^{-\lambda}\right)
$$


is holomorphic, admits a meromorphic extension $H_{Q}$ to the complex plane with at most simple poles at $\lambda=m_{0}+N-j, j \in \mathbb{N}_{0}$, and from (30) we see that $\widehat{\operatorname{Tr}}_{\partial}(A)$ coincides with the regularized value of $H_{Q}$ at $\lambda=0$. Exactly as in Proposition[7.6] we see that we have $\widehat{\operatorname{Tr}}_{\partial}\left(A Q^{-\lambda}\right)=\overline{\operatorname{Tr}}\left(\widetilde{B(\lambda)}_{-(n+1)}\right)$ where the residue class of $A Q^{-\lambda} \in$ $\Psi_{\Phi}^{m_{0}-\lambda,-(n+1)}(X)$ modulo $\Psi_{\Phi}^{\mathbb{Z},-\infty}(X)$ corresponds to $\sum_{k \leq-(n+1)} \varrho_{\mathrm{ff}_{\Phi}}^{-k} \widetilde{B(\lambda)_{k}}$ with $\widetilde{B(\lambda)_{k}} \in \widetilde{\Psi}_{\operatorname{sus}\left({ }^{\Phi} N^{*} Y\right)-\varphi}^{m_{0}-\lambda}(\partial X)$. Now, $\widetilde{B(\lambda)}-(n+1)=\widetilde{\mathcal{N}}_{\Phi}^{(-(n+1))}(A) \widetilde{\mathcal{N}}_{\Phi}^{(0)}(Q)^{-\lambda}=$ $\widetilde{A}_{-(n+1)} Q_{\partial X}^{-\lambda}$, and a further look at the definition of $\overline{\operatorname{Tr}}_{Q_{\partial X}}\left(\widetilde{A}_{-(n+1)}\right)$ completes the proof.

Let us point out that the expression for $\widehat{\operatorname{Tr}}_{\partial}(A)$ for $A \in \Psi_{\Phi}^{m, k}(X)$ with arbitrary $k \in \mathbb{Z}$ is more complicated.

\section{Computation of the indeX}

Recall from 23 that a fibered cusp operator $A \in \Psi_{\Phi}^{p, k}(X)$ is Fredholm between appropriate weighted Sobolev spaces if and only if its $\Phi$-principal symbol ${ }^{\Phi} \sigma^{(p, k)}(A)$ and its normal symbol $\mathcal{N}_{\Phi}^{(k)}(A)$ are both invertible. Let $A$ be such an operator. Then $A$ is invertible modulo $\Psi_{\Phi}^{-1,-1}(X)$, and by a formal Neumann series argument there exists $B \in \Psi_{\Phi}^{-p,-k}(X)$ such that $P_{1}:=\mathrm{id}-B A$ and $P_{2}:=\mathrm{id}-A B$ both belong to $\Psi_{\Phi}^{-\infty,-\infty}(X)$, and, thus, are of trace class. With a little bit more effort and using a formula for the relative inverse of an operator with closed range which goes back at least as far as [41] (see also [8] and [11. Bemerkung 5.7]) we can actually find $B \in \Psi_{\Phi}^{-p,-k}(X)$ such that $P_{1}$ and $P_{2}$ are the orthogonal projections onto the kernel and cokernel of $A$.

Note that $A \otimes B$ then defines a Hochschild cycle in $H H_{1}\left(\Psi_{\Phi}^{\mathbb{Z}, \mathbb{Z}} / \Psi_{\Phi}^{-\infty,-\infty}\right)$. This simple but crucial observation was made by Melrose and Nistor [34, in the context of scalar cusp pseudodifferential operators. An analogous statement holds for any other algebra where elliptic operators admit pseudo-inverses. Melrose and Nistor extend the definition of this 1-cycle to operators acting on sections of a bundle $\mathcal{E}$. By Morita invariance, the homology of such an algebra is isomorphic to the homology of the scalar algebra. Then the boundary map of the long exact sequence of the pair $\left(\Psi_{\Phi}^{-\infty,-\infty}, \Psi_{\Phi}^{\mathbb{Z}, \mathbb{Z}}\right)$ applied to this cycle is a 0-Hochschild class of trace equal to the index of $A$.

A slight extension of this construction is carried out in [35] for a fully elliptic operator between sections of arbitrary vector bundles $\mathcal{E}, \mathcal{F}$. Replace the cycle $A \otimes B$ with $\operatorname{Tr}\left(i_{1} A P_{2} \otimes i_{2} B P_{1}\right)$, where $P_{1}, P_{2}$ are projections from a trivial bundle $\mathbb{C}^{q}$ to $\mathcal{E}, \mathcal{F}, i_{1}, i_{2}$ are embeddings in $\mathbb{C}^{q}$, and $\operatorname{Tr}$ is the generalized trace functional that induces Morita invariance, $\operatorname{Tr}\left(\left(a_{i j}\right) \otimes\left(b_{i j}\right)\right)=\sum a_{i j} \otimes b_{j i}$. Of course, Morita invariance does not apply in this general case, since operators from $\mathcal{E}$ to $\mathcal{F}$ do not form an algebra.

Now we formally follow the computation from [34 modified in [16] and applied in a similar situation in [14, and get the following result. The proof is included for the benefit of the reader.

Proposition 8.1. The boundary map $\delta$ in the long exact sequence in Hochschild homology induced by the short exact sequence

$$
0 \longrightarrow \Psi_{\Phi}^{-\infty,-\infty} \longrightarrow \Psi_{\Phi}^{\mathbb{Z}, \mathbb{Z}} \longrightarrow \Psi_{\Phi}^{\mathbb{Z}, \mathbb{Z}} / \Psi_{\Phi}^{-\infty,-\infty} \longrightarrow 0
$$


applied to a cycle $c \in C_{1}\left(\Psi_{\Phi}^{\mathbb{Z}, \mathbb{Z}} / \Psi_{\Phi}^{-\infty,-\infty}\right)$ is given by

$$
\mathbb{C} \ni \operatorname{Tr}(\delta(c))=\widehat{\operatorname{Tr}}_{\partial}\left(f_{\log \varrho N} c\right)-\widehat{\operatorname{Tr}}_{\sigma}\left(e_{\log Q} c\right) .
$$

Here $f_{\log \varrho_{N}}$ and $e_{\log \varrho_{N}}$ are actions of the derivation $\left[\cdot, \log \varrho_{N}\right]$ on Hochschild 1-chains, namely

$$
\begin{aligned}
& f_{\log \varrho_{N}}\left(A_{0} \otimes A_{1}\right)=A_{0}\left[A_{1}, \log \varrho_{N}\right], \\
& e_{\log \varrho_{N}}\left(A_{0} \otimes A_{1}\right)=\left[A_{0}, \log \varrho_{N}\right] A_{1} .
\end{aligned}
$$

Proof. Let $C$ be a lift of $c$ to $C_{1}\left(\Psi_{\Phi}^{\mathbb{Z}, \mathbb{Z}}\right)$. Then, from the definitions, we have $\delta(c)=$ $[b(C)]_{H H_{0}\left(\Psi_{\Phi}^{-\infty,-\infty}\right)}$, and hence

$$
\operatorname{Tr}(b(C))=\operatorname{Tr}\left(b(C) \varrho_{N}^{z} Q^{-\lambda}\right)_{z=0, \lambda=0} .
$$

To avoid introducing heavy algebraic notation, write formally $C=U \otimes V$. Then

$$
\begin{aligned}
\delta(c)= & \left.\operatorname{Tr}\left((U V-V U) \varrho_{N}^{z} Q^{-\lambda}\right)\right)_{z=0, \lambda=0} \\
= & \left.\operatorname{Tr}\left[\left(U\left(V-\varrho_{N}^{z} V \varrho_{N}^{-z}\right) \varrho_{N}^{z} Q^{-\lambda}\right)+\left(Q^{\lambda} V Q^{-\lambda}-V\right) U \varrho_{N}^{z} Q^{-\lambda}\right)\right]_{z=0, \lambda=0} \\
= & \operatorname{Tr}\left[z U\left(\left[V, \log \varrho_{N}\right]+z F_{1}(z)\right) \varrho_{N}^{z} Q^{-\lambda}\right. \\
& \left.\quad+\lambda\left([\log Q, V]+\lambda F_{2}(\lambda)\right) U \varrho_{N}^{z} Q^{-\lambda}\right]_{z=0, \lambda=0},
\end{aligned}
$$

where $F_{1}(z), F_{2}(\lambda)$ are entire families of operators of fixed order. By Proposition 7.2 the result follows.

There exist more or less canonical choices for $B$ and $Q$. More precisely, choose $B$ to be an inverse of $A$ up to $\Psi_{\Phi}^{-\infty,-\infty}$ and $Q=\left(x^{k} A^{*} A x^{k}\right)^{1 / 2 p}$ modulo $\Psi_{\Phi}^{-\infty,-\infty}$. With these definitions, $\overline{A S}(A):=-\widehat{\operatorname{Tr}}_{\sigma}([A, \log Q] B)$ and $\widehat{\operatorname{Tr}}_{\partial}\left(A\left[B, \log \varrho_{N}\right]\right)$ are independent of choices. Proposition 8.1 implies the following formula for the index of $A$ :

\section{Theorem 8.2.}

$$
\operatorname{index}(A)=\overline{A S}(A)+\widehat{\operatorname{Tr}}_{\partial}\left(A\left[B, \log \varrho_{N}\right]\right) .
$$

The local term can be identified with the coefficient of $t^{0}$ in the asymptotic expansion of the heat kernel of $A^{*} A, A A^{*}$ (this is a universal local expression in the full symbol of $A$, and so we do not need to construct the heat kernel of $\Phi$-operators in order to define it). We are able to determine the non-local term $\widehat{\operatorname{Tr}}_{\partial}\left(A\left[B, \log \varrho_{N}\right]\right)$ explicitly in geometric terms for certain differential operators on manifolds whose boundaries fiber over the circle $S^{1}$.

Fix a connection in the boundary fibration, i.e., a rule for lifting horizontal vector fields. Fix a product $\Phi$-metric on $X$, i.e., a smooth metric on the interior of $X$ that with respect to local product coordinates close to the boundary looks like

$$
\frac{d x^{2}}{x^{4}}+\frac{d \sigma^{2}}{x^{2}}+g
$$

where $\sigma$ is the variable in the circle and $g$ is a family of metrics on the fibers of $\phi$, independent of $x$. Let $E^{ \pm} \rightarrow \partial X$ be vector bundles with fixed Hermitian metrics and compatible connections $\nabla$. Let $\mathcal{E}, \mathcal{F} \rightarrow X$ be vector bundles such that $\left.\left.\mathcal{E}\right|_{\partial X} \simeq \mathcal{F}\right|_{\partial X} \simeq E^{+} \oplus E^{-}$. Let $A \in \operatorname{Diff}_{\Phi}^{1}(X ; \mathcal{E})$ be an elliptic $\Phi$-differential operator of order 1 which near the boundary (for $x<\epsilon$ ) has the form

$$
A=\sigma(x)\left(x^{2} \partial_{x} I_{2}+\left[\begin{array}{cc}
-i x \tilde{\nabla}_{\partial_{\sigma}} & D^{*} \\
D & i x \tilde{\nabla}_{\partial_{\sigma}}
\end{array}\right]\right),
$$


where $D$ is a family of invertible operators on the fibers of the boundary, $\tilde{\nabla}_{\partial_{\sigma}}=$ $\nabla_{\partial_{\sigma}}+\frac{1}{4} \operatorname{Tr}\left(L_{\partial_{\sigma}} g\right)$, and $\sigma(x)$ is a linear isomorphism of $E^{+} \oplus E^{-}$that depends on $x$. This hypothesis is motivated by the fact that when the manifold $X$ and the fibers of the boundary are spin manifolds so that the spin structures on $X$ and on the fibers are compatible, a twisted $\Phi$-Dirac operator on $X$ with respect to the metric (32) has this form.

Theorem 8.3. Under the above assumptions we have

$$
\operatorname{index}(A)=\overline{A S}(A)-\frac{\lim _{a} \eta\left(\delta_{x}\right)}{2},
$$

where the second term is the adiabatic limit of the eta invariant of the operator

$$
\left[\begin{array}{cc}
-i x \tilde{\nabla}_{\partial_{\sigma}} & D^{*} \\
D & i x \tilde{\nabla}_{\partial_{\sigma}}
\end{array}\right]
$$

on $\partial X$ as $x \rightarrow 0$.

For twisted Dirac operators we identify the local term with the characteristic form $\hat{A}(X) \operatorname{ch}(T)$, whose integral on $X$ is convergent. A related formula has been obtained by Nye and Singer [38] for the Dirac operator on $X=S^{1} \times \mathbb{R}^{3}$, where the boundary fibration is the projection $S^{1} \times S^{2} \rightarrow S^{2}$. The result announced above requires a sophisticated proof that will be given in [13].

\section{Appendix A. Symbolic And Schwartz Sections of vector Bundles}

For the convenience of the reader, we recall briefly the definitions of symbolic sections and Schwartz sections of certain vector bundles. An important notion for that is the radial compactification of a vector bundle [28].

It is easy to check that the radial compactification map

$$
\mathrm{RC}: \mathbb{R}^{N} \longrightarrow S_{+}^{N}:=\left\{\left(\omega_{0}, \omega^{\prime}\right) \in S^{N}: \omega_{0} \geq 0\right\}: z \longmapsto\langle z\rangle^{-1}(1, z)
$$

with $\langle z\rangle:=\sqrt{1+|z|^{2}}$ identifies $\mathbb{R}^{N}$ with the interior of the upper half sphere $S_{+}^{N}$, a compact manifold with boundary. The following basic observation is essential for the definitions of this section.

Proposition A.1. Let $\varrho: S_{+}^{N} \rightarrow \overline{\mathbb{R}}_{+}$be a boundary defining function. The radial compactification map $\mathrm{RC}$ induces via pull-pack isomorphisms $\dot{\mathcal{C}}^{\infty}\left(S_{+}^{N}\right) \rightarrow \mathrm{S}\left(\mathbb{R}^{N}\right)$ and $\varrho^{-m} \mathcal{C}^{\infty}\left(S_{+}^{N}\right) \rightarrow S^{m}\left(\mathbb{R}^{N}\right)$ where $\mathrm{S}\left(\mathbb{R}^{N}\right)$ is the space of Schwartz functions on $\mathbb{R}^{N}$, and $S^{m}\left(\mathbb{R}^{N}\right)$ the space of classical symbols of order $m \in \mathbb{C}$.

Now let $V$ be an $N$-dimensional vector space, and $\bar{V}:=V \uplus(V \backslash\{0\}) / \mathbb{R}_{+}$. We want to introduce a differentiable structure on $\bar{V}$. Choose a linear isomorphism $T: V \rightarrow \mathbb{R}^{N}$, and consider

$$
\bar{T}: \bar{V} \longrightarrow S_{+}^{N}: \zeta \longmapsto \begin{cases}\operatorname{RC}(T v), & \zeta=v \in V, \\ \lim _{t \rightarrow \infty} \operatorname{RC}(T t v), & \zeta=[v] \in(V \backslash\{0\}) / \mathbb{R}_{+} .\end{cases}
$$

Then $\bar{T}$ is a well-defined bijection that gives $\bar{V}$ the structure of a differentiable manifold with boundary, diffeomorphic to $S_{+}^{N}$. Since each $A \in \mathrm{GL}\left(\mathbb{R}^{N}\right)$ induces a 
diffeomorphism $\Phi_{A}: S_{+}^{N} \rightarrow S_{+}^{N}$ such that the following diagram commutes:



the differentiable structure on $\bar{V}$ does not depend on the choice of the map $T$.

Everything obviously varies smoothly with parameters; thus, the same construction carries over to smooth vector bundles. Let $X$ be a compact manifold with corners, and $\pi: V \rightarrow X$ a smooth vector bundle of rank $N$. Then there is a unique differentiable structure on $\bar{V}:=V \uplus(V \backslash\{0\}) / \mathbb{R}_{+}$such that $\bar{\pi}: \bar{V} \rightarrow X$ becomes a smooth fiber bundle; for each $q \in X$, the fiber $\bar{\pi}^{-1}(q)$ is obtained by radially compactifying the fiber $\pi^{-1}(q)$. The bundle $\bar{V}$ is called the radial compactification of $V$, whereas the boundary component $\mathbb{S}(V):=(V \backslash\{0\}) / \mathbb{R}_{+}$of $\bar{V}$ is said to be the sphere bundle of $V$.

Now let $E \rightarrow X$ be another vector bundle over $X$. Note that there are canonical embeddings RC $: V \rightarrow \bar{V}$ and $\mathrm{RC}^{*}: \pi^{*} E \rightarrow \bar{\pi}^{*} E$ such that the following diagram commutes:

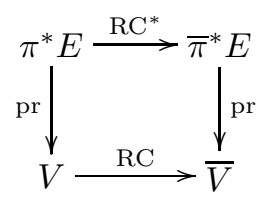

Definition A.2. Let $\varrho_{\sigma}: \bar{V} \rightarrow \overline{\mathbb{R}}_{+}$be a defining function for the boundary face $\mathbb{S}(V)$ of $\bar{V}$. A section $s: V \rightarrow \pi^{*} E$ is said to be a $S$ chwartz (resp. classical) symbol of order $m \in \mathbb{C}$ provided it is the pull-back under RC of a section $\bar{s}: \bar{V} \rightarrow \bar{\pi}^{*} E$ in $\bigcap_{m \in \mathbb{Z}} \varrho_{\sigma}^{-m} \mathcal{C}^{\infty}\left(\bar{V}, \bar{\pi}^{*} E\right)$ (resp. in $\varrho_{\sigma}^{-m} \mathcal{C}^{\infty}\left(\bar{V}, \bar{\pi}^{*} E\right)$ ). We write $\mathrm{S}\left(V, \pi^{*} E\right)$ (resp. $S^{m}\left(V, \pi^{*} V\right)$ ) for the space of all Schwartz functions (resp. symbols) of order $m$. Moreover, in the paper we use the space $S^{\mathbb{Z}}\left(V, \pi^{*} E\right):=\bigcup_{m \in \mathbb{Z}} S^{m}\left(V, \pi^{*} E\right)$ of all symbols of integral order.

\section{Appendix B. Homology of topological filtered Algebras}

Let $A$ be an algebra. The Hochschild complex of $A$ (with coefficients in $A$ ) is defined by $C_{k}(A):=A^{\otimes(k+1)}$ with differential $d: C_{k}(A) \rightarrow C_{k-1}(A)$ given by

$$
\begin{aligned}
b\left(a_{0} \otimes \ldots \otimes a_{k}\right):= & a_{0} a_{1} \otimes \ldots \otimes a_{k}-a_{0} \otimes a_{1} a_{2} \otimes \ldots \otimes a_{k}+\ldots \\
& +(-1)^{k-1} a_{0} \otimes \ldots \otimes a_{k-1} a_{k}+(-1)^{k} a_{k} a_{0} \otimes \ldots \otimes a_{k-1} .
\end{aligned}
$$

If $A$ is a topological algebra, it is more meaningful to involve the topology in the definition (see [6]). Thus, assuming that $A$ is a nuclear Fréchet algebra, one replaces the algebraic tensor product with the projective (completed) tensor product. The most useful example is when $A$ is the algebra of smooth functions on a compact manifold $M$. Then the classical Hochschild-Kostant-Rosenberg map

$$
\begin{aligned}
H K R: C_{k}\left(C^{\infty}(M)\right) & \rightarrow \Lambda^{k}(M), \\
a_{0} \otimes \ldots \otimes a_{k} & \mapsto a_{0} d\left(a_{1}\right) \wedge \ldots \wedge d\left(a_{k}\right),
\end{aligned}
$$

has the property $H K R \circ b=0$ and becomes an isomorphism on the continuous Hochschild homology. This result extends easily to the case where $A$ is the algebra 
of smooth functions on a manifold with boundary or with corners, with Laurent behavior at some boundary hypersurfaces. In that case the continuous Hochschild homology is isomorphic via the $H K R$ map to the space of exterior forms on the manifold, with Laurent behavior at the same hypersurfaces. The map $H K R$ also computes the homology of the field of Laurent series in one variable, the ring of Laurent series in several variables and of polynomial rings; we implicitly assume these facts in the body of the paper.

Since the product in the topological algebra $\Psi_{\Phi}^{\mathbb{Z}, \mathbb{Z}}(X)$ is only separately continuous, we cannot directly apply the definition of continuous Hochschild homology as it is defined for instance in [6]. Instead, we use in a particular case the concept of Hochschild homology for topological filtered algebras formally introduced by Benameur and Nistor [2] to cover exactly the case of pseudodifferential operators. The definition (35) of the chain spaces can be traced back to [4].

Let $\mathcal{A}$ be a bi-filtered algebra with topology with two increasing filtrations $\left\{\mathcal{A}^{i, j}\right\}_{i, j \in \mathbb{Z}}$ adapted to the product structure. The model for $\mathcal{A}$ is $\Psi_{\Phi}^{\mathbb{Z}, \mathbb{Z}}(X)$. So we use the analogous notation for quotients, ideals and associated graded algebras. For example, $\mathcal{A}^{[\mathbb{Z}], \mathbb{Z}}$ means the graded algebra of $\mathcal{A}$ with respect to the first filtration.

Definition B.1. We say that $\mathcal{A}$ is a topological filtered algebra if the following conditions are fulfilled:

(1) $\mathcal{A}=\bigcup_{i, j \in \mathbb{Z}} \mathcal{A}^{i, j}$, and the topology on $\mathcal{A}$ is the strict inductive limit topology (this implies that $\mathcal{A}^{i, j}, \mathcal{A}^{i, \infty}$ and $\mathcal{A}^{\infty, j}$ are closed in $\mathcal{A}$ ).

(2) For each $i, j \in \mathbb{Z}$ the space $\mathcal{A}^{i, j}$ is a nuclear Fréchet space.

(3) The multiplication map $\mathcal{A}^{i_{1}, j_{1}} \otimes \mathcal{A}^{i_{2}, j_{2}} \rightarrow \mathcal{A}^{i_{1}+i_{2}, j ; 1+j_{2}}$ is continuous with respect to the projective topology.

(4) The canonical maps from $\mathcal{A}^{i, j} / \mathcal{A}^{-\infty, j}$ to the projective limit as $k \rightarrow-\infty$ of $\mathcal{A}^{i, j} / \mathcal{A}^{k, j}$, and from $\mathcal{A}^{i, j} / \mathcal{A}^{i,-\infty}$ to the projective limit as $k \rightarrow-\infty$ of $\mathcal{A}^{i, j} / \mathcal{A}^{i, k}$, are isomorphisms of topological vector spaces.

(5) The canonical maps from $\mathcal{A}^{[i], j} / \mathcal{A}^{[i],-\infty}$ to the projective limit as $k \rightarrow-\infty$ of $\mathcal{A}^{[i], j} / \mathcal{A}^{[i], k}$, and from $\mathcal{A}^{i,[j]} / \mathcal{A}^{-\infty,[j]}$ to the projective limit as $k \rightarrow-\infty$ of $\mathcal{A}^{i,[j]} / \mathcal{A}^{k,[j]}$, are isomorphisms of topological vector spaces.

We emphasize that our definition is less general than the one considered in [2].

Since for fixed $i, j$ the product map $\mathcal{A}^{j, j} \otimes \mathcal{A}^{j, j} \rightarrow \mathcal{A}$ is continuous, one defines the continuous Hochschild chain spaces as

$$
C_{k}(\mathcal{A}):=\bigcup_{j \in \mathbb{Z}} \mathcal{A}^{j, j \hat{\otimes}(k+1)} .
$$

Our innovation consists in introducing three filtrations on $C_{k}(\mathcal{A})$, in the following way: a pure tensor $a=a_{0} \otimes \ldots \otimes a_{k}$ is said to belong to the linear space $c_{k}^{i, j ; l}(\mathcal{A})$ if $a_{0} \in \mathcal{A}^{i_{0}, j_{0}}, \ldots, a_{k} \in \mathcal{A}^{i_{k}, j_{k}}$ with $i_{0}+\ldots+i_{k} \leq i, j_{0}+\ldots+j_{k} \leq j$, and such that for any subset $S \subset\{0, \ldots, k\}, \sum_{r \in S} i_{r} \leq l$ and $\sum_{r \in S} j_{r} \leq l$. The space $C_{k}^{i, j ; l}(\mathcal{A})$ is defined as the closure of $c_{k}^{i, j ; l}(\mathcal{A})$ inside $C_{k}(\mathcal{A})$, endowed with the inductive limit

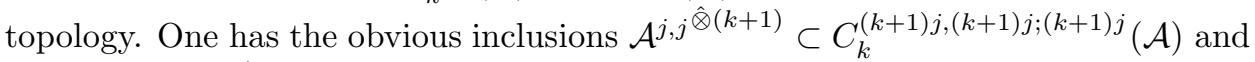
$C_{k}^{i, j ; l}(\mathcal{A}) \subset \mathcal{A}^{l, l} \hat{\otimes}^{(k+1)} ;$ thus

$$
C_{k}(\mathcal{A})=\bigcup_{i, j, l \in \mathbb{Z}} C_{k}^{i, j ; l}(\mathcal{A})
$$


Since the map $b$ is continuous, it extends to the topological chain spaces. The topological Hochschild homology of $\mathcal{A}$ is then defined as the homology of the complex $\left(C_{k}(\mathcal{A}), b\right)$. The advantage of the tri-filtered spaces $C_{k}^{i, j ; l}(\mathcal{A})$ is that

$$
b\left(C_{k}^{i, j ; l}(\mathcal{A})\right) \subset C_{k-1}^{i, j ; l}(\mathcal{A}),
$$

while no analogous property holds for $\mathcal{A}^{j, j} \hat{\otimes}^{(k+1)}$.

Since the filtrations are preserved by $b$, we can form spectral sequences for the homology of the Hochschild complex with respect to these filtrations. A fundamental difficulty appears when analyzing such spectral sequences: the $E^{0}$ terms live on a half-plane. Thus, tautological properties of first-quadrant spectral sequences (like collapsing or convergence) will need careful proofs. In particular, we will need to sum infinite series of chains.

From the definition of topological filtered algebras, every sum $\sum_{q=0}^{\infty} a_{q}, a_{q} \in$ $\mathcal{A}^{k_{1}-q, k_{2}}$ is asymptotically summable in $\mathcal{A}^{k_{1}, k_{2}}$ in the following sense: there exists $a \in \mathcal{A}^{k_{1}, k_{2}}$ such that for all $l \geq 0$ we have

$$
a-\sum_{q=0}^{l} a_{q} \in \mathcal{A}^{k_{1}-l-1, k_{2}} .
$$

The same property also holds for the second filtration. This property implies that every sequence $a_{q} \in C_{k}^{i-q, j ; l}(\mathcal{A}), q \geq 0$, is asymptotically summable inside $C_{k}^{i, j ; l}(\mathcal{A})$. We stress that this statement would fail if we let the third index $l$ vary with $q$.

Now let $\mathcal{A}^{\mathbb{Z}, \mathbb{Z}}$ be a topological filtered algebra such that $\bigcap_{k \in \mathbb{Z}} \mathcal{A}^{k, \mathbb{Z}}=0$. Let ${ }^{\sigma} E(\mathcal{A})$ denote the spectral sequence of the Hochschild complex of $\mathcal{A}$ with respect to the first filtration. We say that ${ }^{\sigma} E(\mathcal{A})$ degenerates at ${ }^{\sigma} E^{p}$ if the higher differentials $d^{p}, d^{p+1}$, etc. all vanish. There exist natural filtrations on Hochschild homology induced from the filtrations of the Hochschild complex; namely, a homology class lives in the first filtration $i$ if it can be represented by a cycle in $C_{*}^{i, \mathbb{Z} ; \mathbb{Z}}(\mathcal{A})$. Moreover, there exist natural edge maps

$$
H H_{i+q}(\mathcal{A})_{[i]} \rightarrow{ }^{\sigma} E_{i, q}^{p}(\mathcal{A})
$$

from the graded group of $H H_{i+q}(\mathcal{A})$ with respect to the filtration into the spectral sequence. We say that ${ }^{\sigma} E(\mathcal{A})$ converges (to the graded Hochschild homology) if it degenerates at ${ }^{\sigma} E^{p}$ for some $p$, if the edge maps (to ${ }^{\sigma} E^{p}$ ) are isomorphisms, and if moreover the "residual" homology $\bigcap_{i \in \mathbb{Z}} H H_{k}(\mathcal{A})_{i}$ vanishes.

Let $\left(C_{*}^{\mathbb{Z}, \mathbb{Z} ; \mathbb{Z}}, d\right)$ be any tri-filtered homology complex, i.e., $C_{*}=\bigcup_{i, j, l \in \mathbb{Z}} C_{*}^{i, j ; l}$. To increase readability of the next paragraphs we will use the following conventions: the subscript $*$ will be generally suppressed. We will work with chains in $C, C[\mathbb{Z}], \mathbb{Z} ; \mathbb{Z}$,

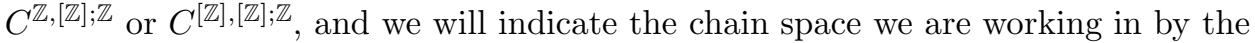
filtration indices: for example, $\alpha \in C^{i,[j] ; l}$ denotes a chain in $C^{\mathbb{Z},[\mathbb{Z}] ; \mathbb{Z}}$. If $\alpha \in C^{i, j ; l}$, we will denote again by $\alpha$ its image in the complex $C^{i,[j] ; l}$.

Lemma B.2. For any spectral sequence associated to a $\mathbb{Z}$-filtered homology complex $\left(C_{*}^{*}, d\right)$ and for all $p \in \mathbb{N}$, the edge maps $H_{i+q}(C)_{[i]} \rightarrow E_{i, q}^{p}$ are injective.

Proof. The upper index in $C_{*}^{*}$ denotes the filtration, which is assumed to be increasing. Let $[\alpha] \in H_{i+k}(C)$ be represented by a cycle $\alpha \in C_{i+k}^{i}$. Assume that the image of $\alpha$ in $E_{i, q}^{p}$ vanishes. Then there exists a class $\left[\beta_{i+p-1}\right] \in E_{i+p-1, i+q+1}^{p-1}$ such that $d^{p-1}\left[\beta_{i+p-1}\right]=[\alpha]_{E^{p-1}}$. This means that there exists a representative $\beta_{i+p-1} \in C^{i+p-1}$ of $\left[\beta_{i+p-1}\right]$ such that $\alpha-d\left(\beta_{i+p-1}\right)$ represents the null 
class at $E^{p-1}$. Inductively, construct $\beta_{i+p-2} \in C^{i+p-2}, \ldots, \beta_{i} \in C^{i}$ such that $\alpha-d\left(\beta_{i+p-1}+\ldots+\beta_{i}\right)$ represents the null class at ${ }^{\sigma} E^{0}$. But this means that $\alpha-d\left(\beta_{i+p-1}+\ldots+\beta_{i}\right) \in C^{i-1}$, or in other words that $[\alpha] \in H_{i+q}(C)_{i-1}$, as claimed.

This lemma is proved using diagram chasing. The essential step in the proof is summing $\beta_{i+p-1}+\ldots+\beta_{i}$ inside $C^{i+p-1}$. As long as the sum is finite, there is of course no problem. However, diagram chasing for other ingredients of convergence will involve summing infinite series of chains of descending filtration orders. This is in principle impossible, since the other filtrations might go up! So we must analyze each step of the diagram chasing very carefully.

Let $N \in \mathbb{Z}$ be fixed. We assume that for any $i, j \in \mathbb{Z}, j^{\prime} \geq j, i^{\prime} \geq i$ and $l \geq 1+\max \left(i+\delta_{0}^{i}, j+\delta_{j}^{0}\right)$, the following assumptions hold for the complex $\bar{C}$ (these will be checked individually for the Hochschild chain complexes of the fibered cusp symbol algebras):

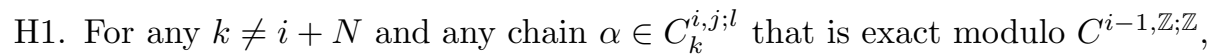
there exists $\beta \in C^{i+1, j+1 ; l}$ such that $b(\beta)+\alpha \in C^{i, j+1 ; l}$ and $b(\beta)+\alpha$ is exact in $C^{[i], j+1 ; l}$.

H2. Let $\alpha \in C_{k}^{i, j ; l}$ be such that $b(\alpha) \in C^{i-2, j ; l}$ and $k \neq i+N$. Then $\alpha$ is exact modulo $C^{i-1, \mathbb{Z} ; \mathbb{Z}}$.

H3. Let $\alpha \in C^{i, j ; l}+C^{i^{\prime}, j-1 ; l}$ be such that $\alpha$ is exact in $C^{i,[j] ; l}$ modulo $C^{i-1,[j] ; l}$. Then there exists $\beta \in C^{i+1, j ; l}$ such that $b(\beta)+\alpha \in C^{i, j ; l}+C^{i^{\prime}, j-1 ; l}$ and $b(\beta)+\alpha$ is exact in $C^{[i],[j] ; l}$.

H4. Let $\alpha \in C^{i, j ; l}+C^{i-1, j^{\prime} ; l}$ be such that $\alpha$ is exact in $C^{[i], j ; l}$. Then there exists $\beta \in C^{i, j ; l}$ such that $b(\beta)+\alpha \in C^{i-1, j^{\prime} ; l}$.

H5. Let $\alpha \in C^{i, j ; l}+C^{i^{\prime}, j-1 ; l}+C^{i-1, j^{\prime} ; l}$ be such that $\alpha$ is exact in $C^{[i],[j] ; l}$. Then there exists $\beta \in C^{i, j ; l}$ such that $b(\beta)+\alpha \in C^{i-1, j^{\prime} ; l}+C^{i^{\prime}, j-1 ; l}$.

H6. $\bigcap_{i \in \mathbb{Z}} C^{i, j ; l}=0$, and any series $\sum_{i=i_{0}}^{-\infty} a_{i}$ with $a_{i} \in C_{k}^{i, j ; l}$ for fixed $j, k, l$ is asymptotically summable.

The assumptions $\mathrm{H} 1$ and $\mathrm{H} 2$ say slightly more than the fact that ${ }^{\sigma} E(C)$ degenerates at ${ }^{\sigma} E^{2}$ and that ${ }^{\sigma} E_{i, *}^{2}=0$ for $* \neq N$. Assumption $\mathrm{H} 3$ is slightly stronger than the degeneracy of ${ }^{\sigma} E\left(C^{\mathbb{Z},[\mathbb{Z}] ; \mathbb{Z}}\right)$ at ${ }^{\sigma} E^{2}$. Assumption $\mathrm{H} 4$ improves the degeneracy of ${ }^{\partial} E\left(\mathcal{A}^{[\mathbb{Z}], \mathbb{Z}}\right)$ at ${ }^{\partial} E^{1}$. Finally, $\mathrm{H} 5$ gives uniform bounds in the third filtration for exact chains. We remark here that this condition and a statement analogous to the second part of Lemma B.7 must be checked in the proof of the convergence result of 4 .

Proposition B.3. Assume that $\mathrm{H} 1-\mathrm{H} 6$ hold for the complex $C$. Let $\alpha \in C_{k}^{i, j ; l}$ be

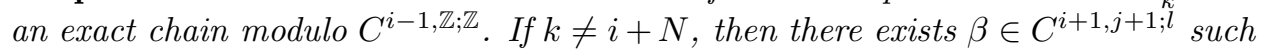
that $b(\beta)+\alpha \in C^{i-1, j ; l}$. If $k=i+N$, then the same conclusion remains valid with possibly higher indices $j, l$.

Proof. Assume first that $k \neq i+N$. By H1 there exists $\beta_{0} \in C^{i+1, j+1 ; l}$ such that $b\left(\beta_{0}\right)+\alpha \in C^{i, j+1 ; l}$ and $b\left(\beta_{0}\right)+\alpha$ is exact in $C^{[i], j+1 ; l}$. By H4, choose $\beta_{1}^{\prime} \in C^{i, j+1 ; l}$ such that $b\left(\beta_{0}+\beta_{1}^{\prime}\right)+\alpha \in C^{i-1, j+1 ; l}$. Assume inductively that for $0 \leq p \leq q-1$ we have found $\beta_{p} \in C^{i-p+1, j+1 ; l}$ and $\beta_{q}^{\prime} \in C^{i-q+1, j+1 ; l}$ such that

$$
b\left(\beta_{0}+\ldots+\beta_{q-1}+\beta_{q}^{\prime}\right)+\alpha \in C^{i-q, j+1 ; l}+C^{i-1, j ; l} .
$$


The initial step $q=1$ was done above. Since $\alpha$ vanishes in $C^{i-q,[j+1] ; l}$, it follows that $b\left(\beta_{0}+\ldots+\beta_{q-1}+\beta_{q}^{\prime}\right)+\alpha$ is exact in $C^{i-q,[j+1] ; l}$. By H3 there exists $\beta_{q}^{\prime \prime} \in C^{i-q+1, j+1 ; l}$ such that $b\left(\beta_{0}+\ldots+\beta_{q-1}+\beta_{q}^{\prime}+\beta_{q}^{\prime \prime}\right)+\alpha \in C^{i-q, j+1 ; l}+C^{i-1, j ; l}$ is exact in $C^{[i-q],[j+1] ; l}$. By $\mathrm{H} 5$ there exists $\beta_{q+1}^{\prime} \in C^{i-q, j+1 ; l}$ such that

$$
b\left(\beta_{0}+\ldots+\beta_{q-1}+\beta_{q}^{\prime}+\beta_{q}^{\prime \prime}+\beta_{q+1}^{\prime}\right)+\alpha \in C^{i-q-1, j+1 ; l}+C^{i-1, j ; l} .
$$

Setting $\beta_{q}:=\beta_{q}^{\prime}+\beta_{q}^{\prime \prime}$, we complete our induction argument.

By H6, the series $\sum \beta_{q}$ is asymptotically summable; so set $\beta:=\sum_{q=0}^{\infty} \beta_{q} \in$ $C_{k+1}^{i+1, j+1 ; l}$. Since

$$
d(\beta)+\alpha \in C^{i-1, j ; l}+\bigcap_{q>0} C^{i-q-1, j+1 ; l}=C^{i-1, j ; l},
$$

using $\mathrm{H} 6$, the conclusion follows for $k \neq i+N$. Assume now that $k=i+N$. The first step of the argument goes through if we allow for possibly higher $\tilde{j}, \tilde{l}$. In the rest of the proof we did not invoke $\mathrm{H} 1$ or $\mathrm{H} 2$. So everything goes through for these new indices $\tilde{j}, \tilde{l}$.

There is something very nontrivial about this proposition. Even without caring about the third index (which is annoying but not serious), a simple application of $\mathrm{H} 1$ would yield a chain $\beta \in C^{i+1, j+1 ; l}$ such that $d(\beta)+\alpha \in C^{i-1, j+1 ; l}$. Then diagram chasing to write $\alpha$ as a boundary would lead to an infinite series of chains of decreasing order in $i$, but increasing in $j$. It is fortunate that we can reduce back the second index in the $\Phi$-case (mainly thanks to the fact that $\mathrm{H} 3$ holds in this case), but we believe that for a multi-fibration pseudodifferential algebra this would not be possible.

Theorem B.4. Let $C$ be a tri-filtered complex satisfying assumptions H1-H6. Then the spectral sequence ${ }^{\sigma} E(C)$ is convergent.

Proof. We would like to invert the edge maps (36). By $\mathrm{H} 1, \mathrm{H} 2$ and $\mathrm{H} 4$ the ${ }^{\sigma} E_{i, *}^{2}$ term is concentrated on the line $*=N$. Then let $A$ be a class at ${ }^{\sigma} E_{i, N}^{2}$. Then $A$ can be represented by a chain $\alpha_{1} \in C_{i+N}^{i, j ; l}$ (note that in the case of the Hochschild complex associated to ${ }^{\Phi} \mathcal{I}_{\sigma}, j$ can be taken $j:=i+N$, see Theorem [5.3). Surviving at ${ }^{\sigma} E^{2}$ means that $b\left(\alpha_{1}\right) \in C_{i+N-1}^{i-1, j ; l}$ and that $b\left(\alpha_{1}\right) \in C_{i+N-1}^{[i-1], j ; l}$ is exact. Thus, by H4 there exists $\alpha_{2} \in C_{i+N}^{i-1, j ; l}$ such that $b\left(\alpha_{2}\right)+b\left(\alpha_{1}\right) \in C_{i+N-1}^{i-2, j ; l}$. Since $(i+N-1)-(i-2) \neq N$ and $b\left(\alpha_{2}+\alpha_{1}\right)$ is exact modulo anything, we can apply Proposition B.3 to get $\alpha_{3} \in C_{i+N}^{i-1, j+1 ; l}$ such that $b\left(\alpha_{1}+\alpha_{2}+\alpha_{3}\right) \in C_{i+N-1}^{i-3, j ; l}$. Inductively, using H4, construct $\alpha_{q} \in C_{i+N}^{i-q+2, j+1 ; l}$ such that $b\left(\alpha_{1}+\ldots+\alpha_{q}\right) \in C_{i+N-1}^{i-q, j ; l}$. By H6, the sum $\alpha:=\sum_{q=1}^{\infty} \alpha_{q} \in C_{i+N}^{i, j ; l}$ is asymptotically summable and represents $A$ at ${ }^{\sigma} E^{2}$ by construction, and

$$
b(\alpha) \in \bigcap_{q \in \mathbb{N}} C_{i+N-1}^{i-q, j ; l}=0 .
$$

So we have found a Hochschild cycle $\alpha$ that maps to $A$ under the edge map (36), which means that the edge maps are isomorphisms (we have seen above that they are injective).

Now we prove the vanishing of the "residual homology" $\bigcap_{i \in \mathbb{Z}} H H_{k}(\mathcal{A})_{i}$. In fact, we prove directly that $H H_{k}(\mathcal{A})_{[k-N-1]}=0$. Let $[\alpha]$ be a homology class represented by a cycle $\alpha \in C^{k-N-1, j ; l}$. By H2 and Proposition B.3 there exists $\beta_{1} \in C^{k-N, j+1 ; l}$ 
such that $b\left(\beta_{1}\right)+\alpha \in C^{k-N-2, j ; l}$. Inductively, there exists $\beta_{q} \in C^{k-N-q+1, j+1 ; l}$ such that $b\left(\beta_{1}+\ldots+\beta_{q}\right)+\alpha \in C^{k-N-q-1, j ; l}$. The sum $\beta:=\sum_{q=1}^{\infty} \beta_{q}$ is summable in $C^{k-N, j+1 ; l}$, and moreover

$$
b(\beta)+\alpha \in \bigcap_{q \in \mathbb{N}} C^{k-N-q-1, j ; l}=0,
$$

which shows that $\alpha$ is exact.

Remark B.5. Proposition B.3 remains valid if we allow any number of exceptional $N$ 's in $\mathrm{H} 1$ and $\mathrm{H} 2$, with the obvious modifications. Thus, Theorem B.4 holds if we allow a finite number of such exceptional $N$ 's. This is needed, for instance, for convergence in the case of the adiabatic algebra [35].

We are now going to establish some sufficient conditions for a topological filtered algebra and the associated symbolic spectral sequence to satisfy the conditions $\mathrm{H} 3$, $\mathrm{H} 4$, and H5. So for the rest of this section we assume that $\mathcal{A}^{[\mathbb{Z}], \mathbb{Z}}$ is isomorphic as an algebra to $\mathbb{C}\left[\rho, \rho^{-1}\right] \otimes A$, where $A$ is one of the algebras $\mathcal{L} \otimes \mathcal{A}^{[0],[0]}$ or $\mathcal{C}^{\infty}(Y)\left[x^{-1}\right]$, for $Y$ a compact manifold with boundary and $x$ a boundary-defining function for $\partial Y$.

Lemma B.6. Properties $\mathrm{H} 4$ and $\mathrm{H} 5$ are satisfied by $\mathcal{A}$ under the above assumption.

Proof. The algebra $\mathcal{A}^{[\mathbb{Z}],[\mathbb{Z}]}$ is isomorphic to the tensor product $\mathcal{A}^{[0],[0]} \otimes \mathbb{C}\left[\rho, \rho^{-1}\right] \otimes$ $\mathbb{C}\left[x, x^{-1}\right]$. By the Eilenberg-Zilber theorem, we can replace the Hochschild complex of $\mathcal{A}^{[\mathbb{Z}],[\mathbb{Z}]}$ by the tensor product of the Hochschild complexes $C_{*}\left(\mathcal{A}^{[0],[0]}\right) \otimes$ $C_{*}\left(\mathbb{C}\left[\rho, \rho^{-1}\right]\right) \otimes C_{*}\left(\mathbb{C}\left[x, x^{-1}\right]\right)$, since the explicit quasi-isomorphisms between the two complexes (the Alexander-Whitney map and the shuffle product) preserve the two gradings and the third filtration index $l$. It is then enough to prove the statement for the algebra $\mathbb{C}\left[x, x^{-1}\right]$ itself. This is done by writing an explicit homotopy between the Hochschild complex $C_{*}\left(\mathbb{C}\left[x, x^{-1}\right]\right)$ and the so-called small complex (see 20, Section 3.1]). Hence we get H5. Property H4 is proved in exactly the same way, by using formal Laurent series rather than Laurent polynomials in the variable $x^{-1}$.

Note now that the algebra $\mathcal{A}^{[\mathbb{Z}], \mathbb{Z}}$ and hence also $H H_{*}\left(\mathcal{A}^{[\mathbb{Z}], \mathbb{Z}}\right)$ have two gradings (depending on choices), given by the negative of the total degrees in $\rho$ and $x$.

Lemma B.7. • Let $[\alpha] \in{ }^{\sigma} E_{i, k}^{1}\left(\mathcal{A}^{\mathbb{Z},[\mathbb{Z}]}\right)$ be a class that corresponds to a class of degree $(i, j)$ when seen as an element of $H H_{i+k}\left(\mathcal{A}^{[\mathbb{Z}],[\mathbb{Z}]}\right)$. Then there exists $\alpha \in$ $C_{i+k}^{i, j ; \max \left(i+\delta_{0}^{i}, j+\delta_{j}^{0}\right)}\left(\mathcal{A}^{\mathbb{Z},[\mathbb{Z}]}\right)$ representing $[\alpha]$.

- Let $[\alpha] \in{ }^{\sigma} E_{i, k}^{1}\left(\mathcal{A}^{\mathbb{Z}, \mathbb{Z}}\right)$ be a class that corresponds to a class of degree $(i, j)$ when seen as an element of $H H_{i+k}\left(\mathcal{A}^{[\mathbb{Z}], \mathbb{Z}}\right)$. Then there exists $\alpha \in C_{i+k}^{i, j ; \max \left(i+\delta_{0}^{i}, j+\delta_{j}^{0}\right)}\left(\mathcal{A}^{\mathbb{Z},[\mathbb{Z}]}\right)$ representing $[\alpha]$.

Proof. It is enough to prove the statement for the algebras $C^{\infty}(Y)\left[x^{-1}\right], \mathcal{L}$ and $\mathbb{C}\left[\rho, \rho^{-1}\right]$ (which is obvious), and then to use the shuffle map to the Hochschild complex of the tensor product algebra.

Corollary B.8. Assume that the spectral sequence ${ }^{\sigma} E\left(\mathcal{A}^{\mathbb{Z},[\mathbb{Z}]}\right)$ degenerates at ${ }^{\sigma} E^{2}$. Then condition $\mathrm{H} 3$ is fulfilled. 
Proof. Let $\alpha \in C^{i, j ; l}$ be such that $\alpha$ is exact in $C^{i,[j] ; l}$ modulo $C^{i-1,[j] ; l}$. This means there exists $\beta_{p} \in C^{i+p, j ; \mathbb{Z}}$ such that $b(\beta)+\alpha \in C^{i-1,[j] ; \mathbb{Z}}$. Then $\alpha$ survives as the null class in ${ }^{\sigma} E^{p}\left(\mathcal{A}^{\mathbb{Z},[\mathbb{Z}]}\right)$, so by hypothesis also at ${ }^{\sigma} E^{2}$. In other words, there exists $[\beta] \in{ }^{\sigma} E^{1}$ with $d^{1}[\beta]=-[\alpha]$. Clearly $[\beta]$ is of (negative) degree $(i+1, j)$ in $\rho$ and $x$. By Lemma B.7 we can choose a representative $\beta \in C^{i+1,[j] ; l}$ provided $l \geq \max \left(i+1 \delta_{0}^{i}, j+\delta_{j}^{0}\right)$. This is the desired chain $\beta$.

Corollary B.9. Let $\mathcal{A}$ be a topological filtered algebra such that the second filtration is constant. Assume that ${ }^{\sigma} E(\mathcal{A})$ degenerates at ${ }^{\sigma} E^{2}$, that $\mathcal{A}^{[\mathbb{Z}]} \simeq \mathbb{C}\left[\rho, \rho^{-1}\right] \otimes \mathcal{A}^{[0]}$, and that $\mathcal{A}^{[0]}$ is $H$-unital. Then ${ }^{\sigma} E(\mathcal{A})$ is convergent.

Proof. The issue is finding asymptotically summable chains in

$$
C_{k}(\mathcal{A}):=\bigcup_{i, l \in \mathbb{Z}} C_{k}^{i ; l}(\mathcal{A}) .
$$

Because the graded algebra is a tensor product, we see by Lemmas B.6 and B.7 that if $\alpha \in C^{i ; l}$ is exact modulo $C^{i-1 ; \mathbb{Z}}$, then there exists $\beta \in C^{i+1 ; l}$ such that $b(\beta)+\alpha \in C^{i-1 ; l}$, provided $l \geq i+1+\delta_{i}^{0}$. This allows us to conclude that the edge maps are isomorphisms and that the residual homology vanishes, by constructing asymptotically summable series of chains via diagram chasing as in Theorem B.4

The last result is implicitly used in [4].

\section{REFERENCES}

[1] B. Ammann, R. Lauter, V. Nistor, and A. Vasy. Complex powers and non-compact manifolds, math.OA/0211305, preprint, November 2002.

[2] M. Benameur and V. Nistor, Homology of complete symbols and noncommutative geometry, Landsman, N. P. (ed.) et al., Quantization of singular symplectic quotients, Basel, Birkhäuser. Prog. Math. 198 (2001), 21-46.

[3] J.-L. Brylinski, A differential complex for Poisson manifolds, J. Differential Geometry 28 (1988), 93-114. MR 89m:58006

[4] J.-L. Brylinski and E. Getzler, The homology of algebras of pseudodifferential operators and the noncommutative residue, $K$-Theory 1 (1987), 385-403. MR 89j:58135

[5] B. Bucicovschi, An extension of the work of V. Guillemin on complex powers and zeta functions of elliptic pseudodifferential operators, Proc. Amer. Math. Soc. 127 (1999), 3081-3090. MR 2000a:58085

[6] A. Connes, Noncommutative Geometry, Academic Press, New York - London (1994). MR 95j: 46063

[7] A. Connes, Gravity coupled with matter and the foundation of non-commutative geometry, Comm. Math. Phys. 182 (1996), 155-176. MR 98f:58024

[8] H. O. Cordes, On a class of $C^{*}$-algebras, Math. Annalen 170 (1967), 283-313. MR 35:749

[9] Yu. V. Egorov and B.-W. Schulze, Pseudodifferential operators, Singularities, Applications, volume 93 of Operator Theory and Applications. Birkäuser, Basel (1997). MR 98e:35181

[10] C. L. Epstein, R. B. Melrose, and G. A. Mendoza, Resolvent of the Laplacian on strictly pseudoconvex domains, Acta Math. 167 (1991), 1-106. MR 92i:32016

[11] B. Gramsch. Relative Inversion in der Störungstheorie von Operatoren und $\Psi$-Algebren, Math. Annalen 269 (1984), 27-71. MR 86j:47065

[12] V. Guillemin, A new proof of Weyl's formula on the asymptotic distribution of eigenvalues, Adv. in Math. 55 (1985), 131-160. MR 86i:58135

[13] R. Lauter and S. Moroianu. An index formula on manifolds with fibered cusp ends, preprint, 2002.

[14] R. Lauter and S. Moroianu, Homology of pseudo-differential operators on manifolds with fibered boundaries, Journal Reine Angew. Math. 547 (2002), 207-234. 
[15] R. Lauter and S. Moroianu, The index of cusp operators on manifolds with corners, Ann. Global Anal. Geom. 21, nr. 1 (2002), 31-49. MR 2003e:58033

[16] R. Lauter and S. Moroianu, On the index formula of Melrose and Nistor. Preprint Nr. 3, IMAR, Bucharest, March 2000.

[17] R. Lauter and S. Moroianu, Fredholm theory for degenerate pseudodifferential operators on manifolds with fibered boundaries, Comm. Partial Differential Equations 26 (2001), 233-283. MR 2002e:58052

[18] R. Lauter and V. Nistor, Analysis of geometric operators on open manifolds: a groupoid approach, In N.P. Landsman, M. Pflaum, and M. Schlichenmaier, ed., Quantization of Singular Symplectic Quotients, vol. 198 of Progress in Mathematics, pp. 181-229. Birkhäuser, Basel Boston - Berlin, 2001.

[19] M. Lesch and M. J. Pflaum, Traces on algebras of parameter dependent pseudodifferential operators and the eta-invariant, Trans. Amer. Math. Soc. 352 (2000), 4911-4936. MR 2001b:58042

[20] J.-L. Loday, Cyclic homology, volume 301 of Grundlehren der Mathematischen Wissenschaften, Springer-Verlag, Berlin - Heidelberg - New York (1992). MR 94a:19004

[21] F. Mantlik, Norm closure and extension of the symbolic calculus for the cone algebra, Ann. Global Anal. and Geometry 13 (1995), 339-376. MR 97a:58183

[22] R. R. Mazzeo, Elliptic theory of differential edge operators I, Comm. Partial Differential Equations 16 (1991), 1615-1664. MR 93d:58152

[23] R. R. Mazzeo and R. B. Melrose, Pseudodifferential operators on manifolds with fibred boundaries, Asian J. Math. 2 (1998), 833-866. MR 2000m:58046

[24] J. McCleary, User's guide to spectral sequences, volume 12 of Mathematical Lecture Series, Publish or Perish, Wilmington (1985). MR 87f:55014

[25] R. B. Melrose, Analysis on manifolds with corners, in preparation.

[26] R. B. Melrose, Pseudodifferential operators, corners and singular limits, In Proceeding of the International Congress of Mathematicians, Kyoto, Springer-Verlag, Berlin - Heidelberg New York (1990), 217-234. MR 93j:58131

[27] R. B. Melrose, The Atiyah-Patodi-Singer index theorem, volume 4 of Research Notes in Mathematics, A. K. Peters, Wellesley, Massachusetts (1993). MR 96g:58180

[28] R. B. Melrose, Spectral and scattering theory for the Laplacian on asymptotically Euclidean space, In M. Ikawa (ed.), Spectral and Scattering Theory, volume 162 of Lecture Notes in Pure and Applied Mathematics, pages 85-130, New York, 1994. Marcel Dekker Inc. Proceedings of the Taniguchi International Workshop held in Sanda, November 1992. MR 95k:58168

[29] R. B. Melrose, The eta invariant and families of pseudodifferential operators, Math. Res. Letters 2 (1995), 541-561. MR 96h:58169

[30] R. B. Melrose, Geometric scattering theory, Cambridge University Press, 1995. MR 96k:35129

[31] R. B. Melrose, Fibrations, compactifications and algebras of pseudodifferential operators, In L. Hörmander and A. Mellin, editors, Partial Differential Equations and Mathematical Physics, Birkhäuser, Boston, 1996, 246-261. MR 98j:58117

[32] R. B. Melrose, Geometric optics and the bottom of the spectrum, In F. Colombini and N. Lerner, editors, Geometrical optics and related topics, volume 32 of Progress in nonlinear differential equations and their applications, Birkhäuser, Basel - Boston - Berlin (1997).

[33] R. B. Melrose and V. Nistor, Higher index and $\eta$-invariants for suspended algebras of pseudodifferential operators, unfinished manuscript.

[34] R. B. Melrose and V. Nistor, Homology of pseudodifferential operators I. Manifolds with boundary, to appear in Amer. Math. J., Preprint, May 1996.

[35] S. Moroianu, Higher residues on the algebra of adiabatic pseudodifferential operators, Ph.D. thesis, Massachusetts Institute of Technology, Cambridge, Massachusetts (1999).

[36] W. Müller, Manifolds with cusps of rank one. Spectral theory and $L^{2}$-index theorem, Lecture Notes in Mathematics 1244, Springer-Verlag, Berlin (1987). MR 89g:58196

[37] R. Nest and E. Schrohe, Hochschild homology of Boutet de Monvel's algebra, in preparation.

[38] T. M. W. Nye and M. A. Singer, An $L^{2}$-index theorem for Dirac operators on $S^{1} \times \mathbb{R}^{3}$, J. Funct. Anal. 177 (2000), 203-218. MR 2002a:58020

[39] V. Nistor, Groupoids and the integration of Lie algebroids, J. Math. Soc. Japan 52 (2000), 847-868. MR 2002e:58035] 
[40] S. Rempel and B.-W. Schulze, Complete Mellin and Green symbolic calculus in spaces with conormal asymptotics, Ann. Global Anal. Geometry 4 (1986), 137-224. MR 89f:58132

[41] C. E. Rickart, Banach algebras with an adjoint operation, Ann. Math. 47 (1946), 528-550. MR 8:159b

[42] B. Vaillant, Index- and spectral theory for manifolds with generalized fibered cusps, Ph.D. thesis, University of Bonn (2001).

[43] M. Wodzicki, Cyclic homology of differential operators, Duke Math. J. 54 (1987), 641-647. MR 88k:32035

[44] M. Wodzicki, Noncommutative residue. I. Fundamentals, In $K$-theory, arithmetic and geometry (Moscow, 1984-1986), Springer, Berlin (1987), 320-399. MR 90a:58175

[45] M. Wodzicki, Cyclic homology of pseudodifferential operators and noncommutative Euler class, C. R. Acad. Sci. Paris Sér. I Math. 306 (1988), 321-325. MR 89h:58189

[46] J. Wunsch, Propagation of singularities and growth for Schrödinger operators, Duke Math. J. 98 (1999), 137-186. MR 2000h:58054

Fachbereich 17 - Mathematik, Universität Mainz, D-55099 Mainz, Germany

E-mail address: lauter@mathematik.uni-mainz.de

Institutul de Matematică al Academiei Române, P.O. Box 1-764, RO-70700 Bucharest, ROMANIA

E-mail address: moroianu@alum.mit.edu 